\title{
1 Efficient and precise single-cell reference atlas mapping with Symphony
}

2 Joyce B. Kang ${ }^{1-5}$, Aparna Nathan ${ }^{1-5}$, Fan Zhang ${ }^{1-5}$, Nghia Millard ${ }^{1-5}$, Laurie Rumker ${ }^{1-5}$, D. Branch

3 Moody $^{3}$, llya Korsunsky ${ }^{1-5 * *}$, Soumya Raychaudhuri ${ }^{1-6 * *}$

$4 \quad{ }^{1}$ Center for Data Sciences, Brigham and Women's Hospital, Boston, MA, USA

$5{ }^{2}$ Division of Genetics, Department of Medicine, Brigham and Women's Hospital and Harvard Medical

6 School, Boston, MA, USA

$7 \quad{ }^{3}$ Division of Rheumatology, Inflammation, and Immunity, Department of Medicine, Brigham and

8 Women's Hospital and Harvard Medical School, Boston, MA, USA

$9 \quad{ }^{4}$ Department of Biomedical Informatics, Harvard Medical School, Boston, MA, USA

$10{ }^{5}$ Program in Medical and Population Genetics, Broad Institute of MIT and Harvard, Cambridge, MA, 11 USA

$12{ }^{6}$ Versus Arthritis Centre for Genetics and Genomics, Centre for Musculoskeletal Research, Manchester

13 Academic Health Science Centre, The University of Manchester, Manchester, UK

** These authors jointly supervised this work.

17 Correspondence to:

18 llya Korsunsky

19 Harvard New Research Building

2077 Avenue Louis Pasteur

21 Boston, MA 02115

22 ikorsunskiy@bwh.harvard.edu

24 Soumya Raychaudhuri

25 Harvard New Research Building

2677 Avenue Louis Pasteur, Suite 250

27 Boston, MA 02115

28 soumya@broadinstitute.org

29 Ph: 617-525-4484 Fax: 617-525-4488 


\section{Abstract}

31 Recent advances in single-cell technologies and integration algorithms make it possible to construct

32 comprehensive reference atlases encompassing many donors, studies, disease states, and sequencing

33 platforms. Much like mapping sequencing reads to a reference genome, it is essential to be able to map

34 query cells onto complex, multimillion-cell reference atlases to rapidly identify relevant cell states and

35 phenotypes. We present Symphony (https://github.com/immunogenomics/symphony), an algorithm for

36 building integrated reference atlases of millions of cells in a convenient, portable format that enables

37 efficient query mapping within seconds. Symphony localizes query cells within a stable low-dimensional

38 reference embedding, facilitating reproducible downstream transfer of reference-defined annotations to

39 the query. We demonstrate the power of Symphony by (1) mapping a multi-donor, multi-species query

40 to predict pancreatic cell types, (2) localizing query cells along a developmental trajectory of human

41 fetal liver hematopoiesis, and (3) inferring surface protein expression with a multimodal CITE-seq atlas

42 of memory T cells.

43

44 Keywords: single-cell genomics, scRNA-seq, reference mapping, annotation 


\section{Introduction}

46 Advancements in single-cell RNA-sequencing (scRNA-seq) have launched an era in which individual

47 studies can routinely profile $10^{4}-10^{6}$ cells $^{1-3}$, and multimillion-cell datasets are already emerging ${ }^{4,5}$.

48 Single-cell resolution enables the discovery and refinement of cell states across diverse clinical and

49 biological contexts ${ }^{6-11}$. To date, most studies redefine cell states from scratch, making it difficult to

50 compare results across studies and thus hampering reproducibility. Coordinated large-scale efforts,

51 exemplified by the Human Cell Atlas $(\mathrm{HCA})^{12}$, aim to establish comprehensive and well-annotated

52 reference datasets comprising millions of cells that capture the broad spectrum of cell states. Building

53 these reference atlases requires integrating multiple datasets that may have been collected under

54 different technical and biological conditions. Hence, reference construction requires application of one

55 of many recently developed single-cell integration algorithms ${ }^{13-19}$. Our group previously developed

56 Harmony ${ }^{15}$, a fast, accurate, and well-reviewed method ${ }^{20}$ that is able to explicitly model complex study

57 design, a property that makes it suitable for integrating complex datasets into reference atlases ${ }^{21-24}$.

58 The potential to define common cell states using reference maps has already been demonstrated ${ }^{25,26}$.

59 For example, we built an integrated reference of $\sim 80,000$ single-cell profiles of fibroblasts from human

60 lung, synovium, salivary gland, and intestine and successfully mapped fibroblasts from human skin and

61 mouse synovium, lung, and intestine to analyze conserved states across tissues and species ${ }^{25}$. Once

62 such reference atlases are painstakingly constructed, interpretation of new datasets requires the ability

63 to quickly map single-cell profiles into these reference atlases. This enables interpretation of new

64 datasets by transferring annotations and metadata of interest from nearby reference cells.

Fast mapping of query cells against a large, stable reference is a well-recognized open

66 problem $^{27}$ and active area of research ${ }^{28-30}$. One inefficient but accurate approach to project reference

67 and query cells into a joint embedding is to integrate both sets of cells together de novo, resulting in

68 what might be considered a "gold standard" embedding. While this approach is reasonable for relatively

69 small reference datasets, it is intractable for atlas-sized references with millions of cells. It requires

70 users to "rebuild" the reference for each analysis, which may be computationally challenging and 
71 require administratively cumbersome exchanges of large-scale datasets. Furthermore, de novo

72 integration may corrupt the reference embedding once a reference is carefully constructed and

73 annotated. It is instead preferable to freeze the reference when mapping new query cells onto it.

Here, we define reference mapping to mean placing query cells within the same embedding as integrated reference cells without requiring access to the raw data on all individual reference cells. Importantly, this embedding does not take advantage of any particular annotation, such as cell type labels, which may be refined or updated over time. This is in contrast to automated cell type classifiers, such as scmap ${ }^{31}$, which assign rigid annotations based on reference datasets in a supervised manner. Reference mapping approaches introduced so far include Seurat v $4^{30}$, which is compatible with Seurat integration ${ }^{18}$, and scArches, which is compatible with autoencoders such as scANVI $I^{32}$ and $\operatorname{trVAE}^{33}$.

81 These approaches separate reference building, which integrates datasets in the reference into a lowdimensional embedding, from query mapping, which uses a compressed version of the reference to efficiently map cells into the reference embedding. They further contrast with de novo integration methods like $B B K N N^{34}$, Seurat $\mathrm{v}^{18}$, and Harmony ${ }^{17}$, which enable reference building but are slow and require access to the raw data and batch information on individual reference cells. High-quality reference mapping requires both a framework to efficiently store an integrated reference, and a fast and accurate procedure to map query datasets.

An ideal reference mapping algorithm must meet four key requirements. First, similar to de novo integration algorithms, they must be able to remove confounding signals due to complex study design in both the reference and query. In addition, they must be able to scale to large datasets, map with high

91 accuracy, and enable inference of diverse query cell annotations based on reference cells. We present Symphony, a novel algorithm to compress a large, integrated reference and map query cells to a precise location in the reference embedding within seconds. Through multiple real-world dataset analyses, we show that Symphony can enable accurate downstream inference of cell type, developmental trajectory position, and protein expression, even when the query itself contains complex confounding technical and biological effects. 


\section{Results}

\section{Symphony compresses an integrated reference for efficient query mapping}

Symphony comprises two main algorithms: reference compression and mapping (Methods, Fig. S1a).

Symphony reference compression captures and structures information from multiple reference datasets

101 into an integrated and concise format that can subsequently be used to map query cells (Fig. 1a-b).

102 Symphony builds upon the linear mixture model framework first introduced by Harmony ${ }^{17}$. Briefly, in a 103 low-dimensional embedding, such as principal component analysis (PCA), the model represents cell 104 states as soft clusters, in which a cell's identity is defined by probabilistic assignments across one or 105 more clusters. For de novo integration of the reference, cells are iteratively assigned soft cluster 106 memberships, which are used as weights in a linear mixture model to remove unwanted covariate107 dependent effects. To store the reference efficiently without saving information on individual reference 108 cells, Symphony computes summary statistics learned in the low-dimensional space (Fig. 1b, Methods), returning computationally efficient data structures containing the "minimal reference elements" needed to map new cells. These include the means and standard deviations used to scale

111 the genes, the gene loadings from PCA (or another low dimensional projection, e.g. canonical

112 correlation analysis [CCA]) on the reference cells, soft-cluster centroids from the integrated reference,

113 and two "compression terms" (a $k \times 1$ vector and $k \times d$ matrix, where $k$ is the number of clusters and $d$ is 114 the dimensionality of the embedding) (Methods, Supplementary Equations, Fig. S1b). 


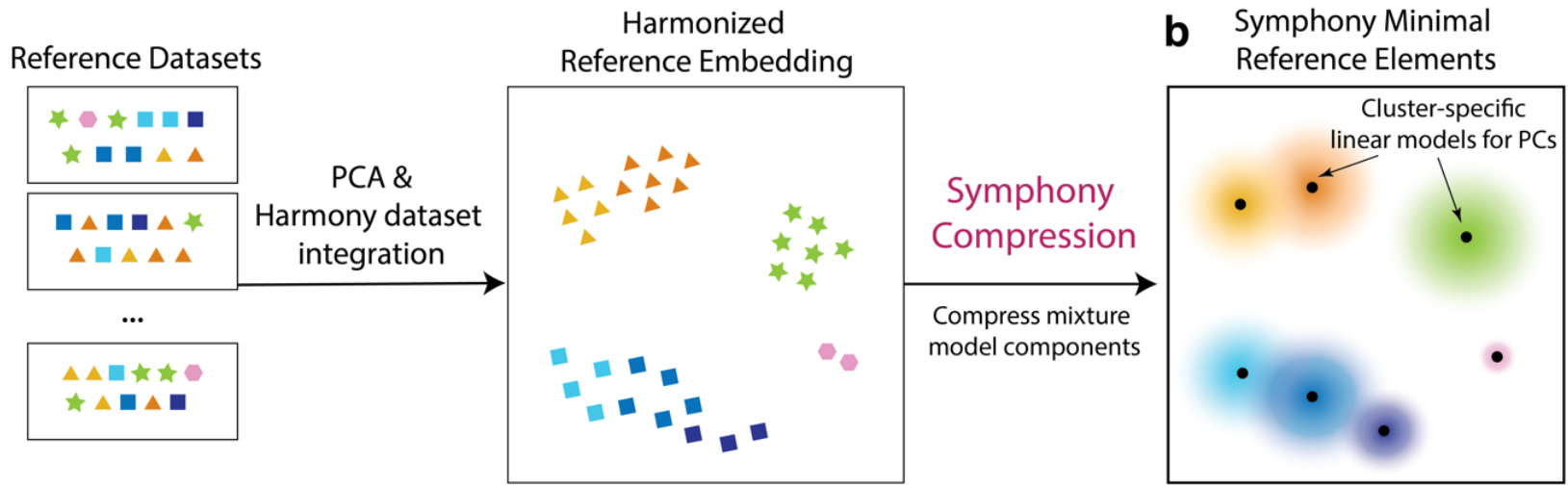

C

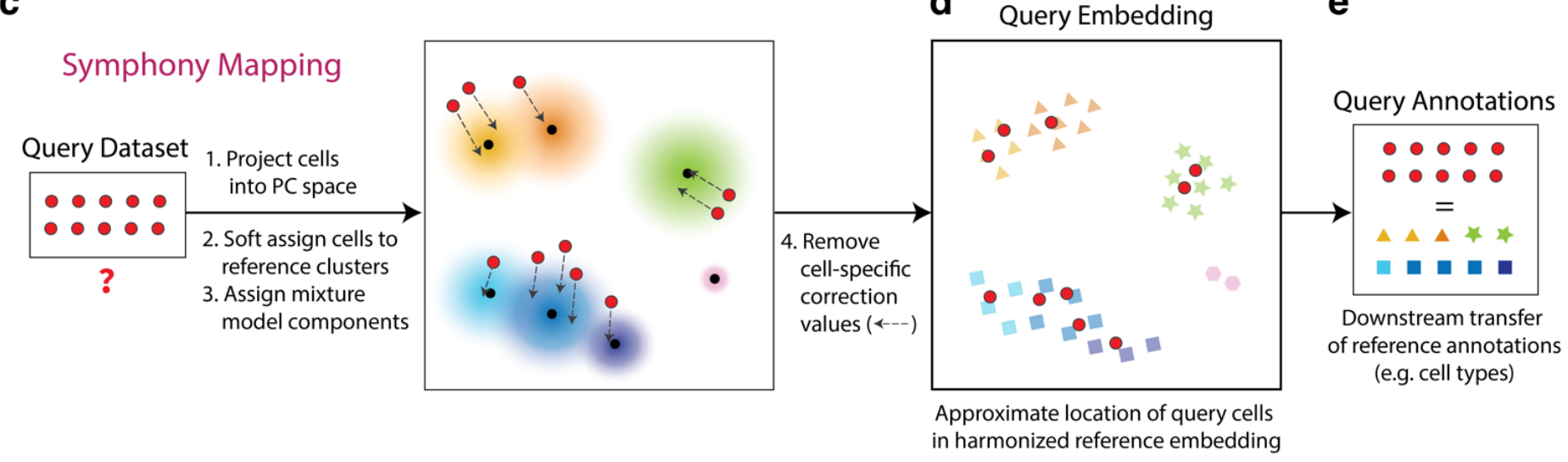

Figure 1. Symphony Overview. Symphony comprises two algorithms: Symphony compression (a-b) and Symphony mapping (c-d). (a) To construct a reference atlas, cells from multiple datasets are embedded in a lower-dimensional space (e.g. PCA), in which dataset integration (Harmony) is performed to remove dataset-specific effects. Shape indicates distinct cell types, and color indicates finer-grained cell states. (b) Symphony compression represents the information captured within the harmonized reference in a concise, portable format based on computing summary statistics for the reference-dependent components of the linear mixture model. Symphony returns the minimal reference elements needed to efficiently map new query cells to the reference. (c) Given an unseen query dataset and compressed reference, Symphony mapping precisely localizes the query cells to their appropriate locations within the integrated reference embedding (d). Reference cell locations do not change during mapping. (e) The resulting joint embedding can be used for downstream transfer of reference-defined annotations to the query cells. See Fig. S1. 
123 components to estimate and regress out the query batch effects (Fig. 1d). Importantly, the reference

124 cell embedding remains stable during mapping. Embedding the query within the reference coordinates 125 enables downstream transfer of annotations from reference cells to query cells, including discrete cell 126 type classifications, quantitative cell states (e.g. position along a trajectory), or expression of missing 127 genes or proteins (Fig. 1e).

\section{Symphony approximates de novo integration of PBMCs without reintegration of}

131 three conditions are met: (I) all cell states represented in the query data set are captured by the

132 reference dataset, (II) the number of query cells is much smaller than the number of reference cells, 133 and (III) the query dataset has a design matrix that is independent of reference datasets (i.e. non134 overlapping batches in reference and query). As the scope of available single-cell atlases continues to 135 grow, it is reasonable to assume that reference datasets are large and all-inclusive, making conditions 136 (I) and (II) well-supported. Condition (III) is also typically met if the query data was generated in 137 separate experiments from the reference.

To demonstrate that Symphony mapping closely approximates running de novo integration on all cells, we applied Symphony to 20,792 peripheral blood mononuclear cells (PBMCs) assayed with three different 10x technologies: 3'v1, 3'v2, and 5'. We performed three mapping experiments. For each, we built an integrated Symphony reference from two technologies, then mapped the third

142 technology as a query. The resulting Symphony embeddings were compared to a gold standard embedding obtained by running Harmony on all three datasets together. Visually, we found that the

144 Symphony embedding for each mapping experiment (Fig. 2a) closely reproduced the overall structure 145 and cell type information of the gold standard embedding (Fig. 2b). To quantitatively assess the 146 degrees of dataset mixing we use the Local Inverse Simpson's Index (LISI) ${ }^{17}$ metric. For a given 147 categorical label assigned to each cell (in this case, technology), LISI indicates the effective number of 148 categories represented in the local neighborhood of each cell; higher LISI scores correspond to better 
a
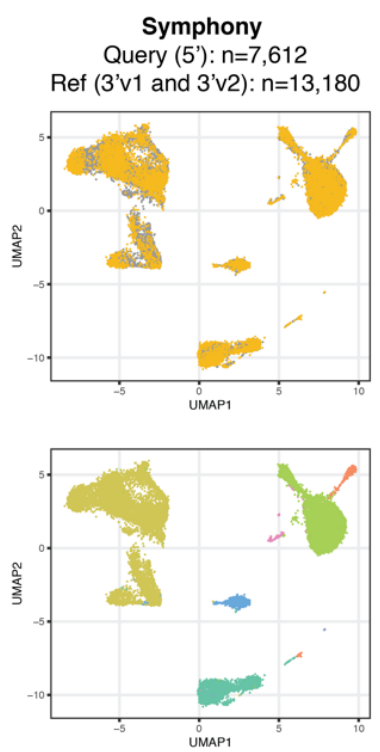

C

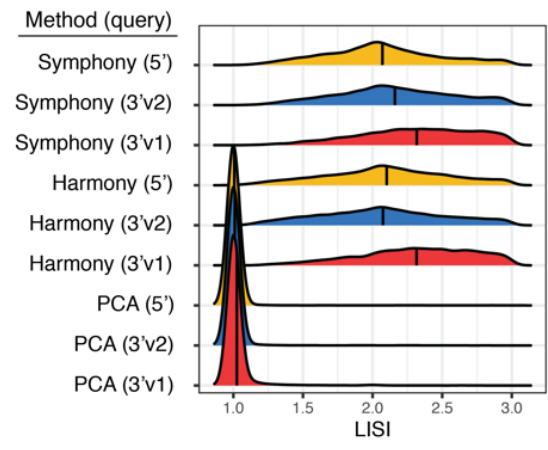

Symphony

Query (3'v2): $n=8,372$ Ref ( $3^{\prime} v 1$ and $\left.5^{\prime}\right): n=12,420$
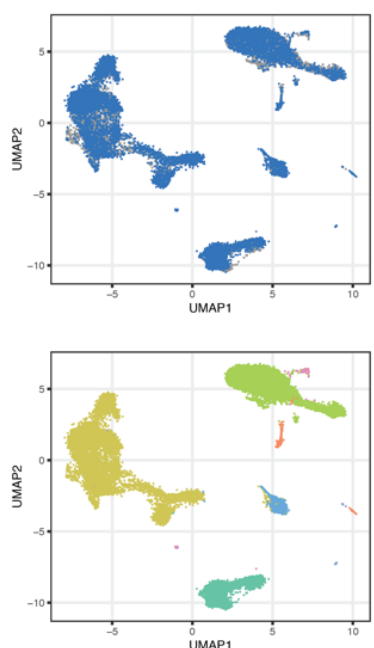

d
Symphony

Query (3'v1): $n=4,808$

Ref ( $3^{\prime} v 2$ and $\left.5^{\prime}\right)$ : $n=15,984$
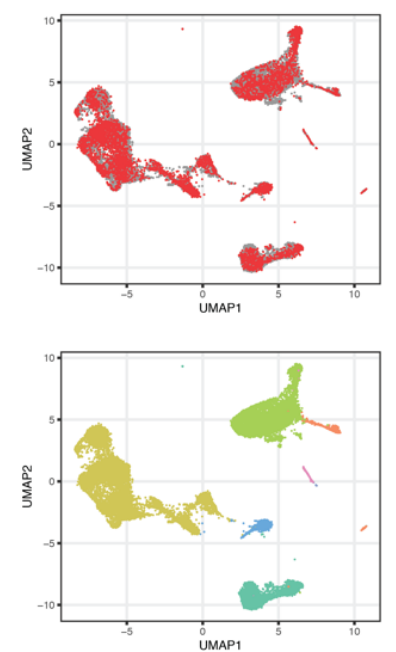

UMAP1

b

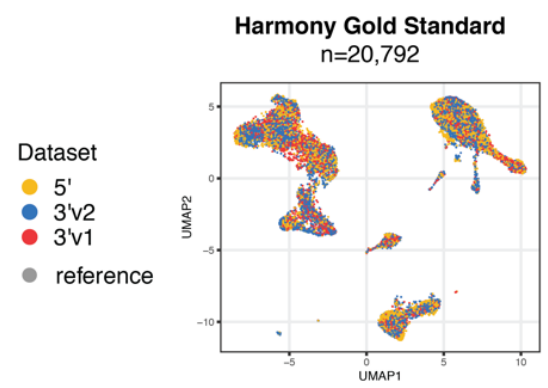

Cell Type
B
DC
HSC
MK
Mono
NK
T

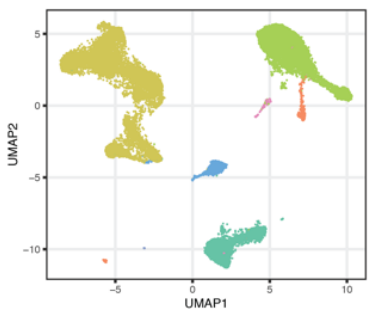

e

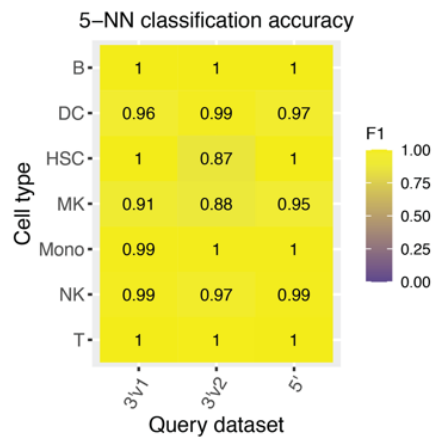

Figure 2. Symphony approximates de novo integration without reintegration of the reference cells. Three PBMC datasets were sequenced with different 10x protocols: 5' (yellow, $n=7,697$ cells), 3'v2 (blue, n=8,380 cells), and 3'v1 (red, $\mathrm{n}=4,809$ cells). We ran Symphony three times, each time mapping one dataset onto a reference built from integrating the other two. (a) Symphony embeddings generated across the three mapping experiments (columns). Top row: cells colored by query (yellow, blue, or red) or reference (gray), with query cells plotted in front. Bottom row: cells colored by cell type: B cell (B), dendritic cell (DC), hematopoietic stem cell (HSC), megakaryocyte (MK), monocyte (Mono), natural killer cell $(\mathrm{NK})$, or $\mathrm{T}$ cell $(\mathrm{T})$, with query cells plotted in front. (b) For comparison, gold standard de novo Harmony embedding colored by dataset (top) and cell type (bottom). (c) Distribution of technology LISI scores for query cell neighborhoods in the Symphony, gold standard, and a standard PCA embeddings on all cells. (d) Distribution of k-NN-corr (Spearman correlation between the similarities between the neighbor-pairs in the Harmony embedding and the similarities between the same neighbor-pairs in the Symphony embedding) across query cells for $k=500$, colored by query dataset. (e) Classification accuracy as measured by cell type F1 scores for query cell type annotation using 5-NN on the Symphony embedding. See Fig. S2. 
149 mixing of cells across batches. LISI scores in Symphony embeddings (mean LISI 2.16, 95\% CI [2.16,

$1502.17]$ ) and de novo integration embeddings (mean LISI 2.14, 95\% CI [2.13, 2.15]) were nearly identical

151 (Fig. 2c, Methods).

To directly assess similarity of the local neighborhood structures, we computed the correlation between the local neighborhood adjacency graphs generated by Symphony and de novo integration.

154 We define a new metric called k-nearest-neighbor correlation (k-NN-corr), which quantifies how well the local neighborhood structure in a given embedding is preserved in an alternative embedding by looking at the correlation of neighbor cells sorted by distance (Fig. S2a-e). Anchoring on each query cell, we calculate (1) the pairwise similarities to its $k$ nearest reference neighbors in the gold standard embedding and (2) the similarities between the same query-reference neighbor pairs in the alternate embedding (Methods), then calculate the Spearman correlation between (1) and (2). k-NN-corr ranges

160 from -1 to +1 , where +1 indicates a perfectly preserved sorted ordering of neighbors. We find that for

$161 k=500$, the Symphony embeddings produce a $k-N N-c o r r ~>0.4$ for $77.3 \%$ of cells (and positive k-NN-corr

162 for $99.9 \%$ of cells), demonstrating that Symphony not only maps query cells to the correct broad cluster 163 but also preserves the distance relationships between nearby cells in the same local region (Fig. 2d).

164 As a comparison, we calculated k-NN-corr for a simple PC projection of the query cells (with no 165 correction step) using the original reference gene loadings prior to integration and observed 166 significantly lower correlations (Wilcoxon signed-rank $p<2.2 e-16$ ), with $k-N N-c o r r ~>0.4$ for $39.9 \%$ of cells 167 (Fig. S2f). Symphony enables accurate cell type classification of PBMCs across technologies

169 If Symphony is effective, then cells should be mapped close to cells of the same cell type, enabling 170 accurate cell type classification. To test this, we performed post-mapping query cell type classification 171 in the 10x PBMCs example from above. We used a 5-NN classifier to annotate query cells across 7 cell 172 types based on the nearest reference cells in the harmonized embedding and compared the predictions 173 to the ground truth labels assigned a priori with lineage-specific marker genes (Methods, Table S2). 174 Across all three experiments, predictions using the Symphony embeddings achieved $99.5 \%$ accuracy 
175 overall, with a median cell type F1-score (harmonic mean of precision and recall, ranging from 0 to 1 ) of

1760.99 (Fig. 2e, Table S3). This indicates that Symphony appropriately localizes query cells in

177 harmonized space to enable the accurate transfer of cell type labels.

Automatic cell type classification represents an open area of research ${ }^{31,35-38}$. Existing supervised classifiers assign a limited set of labels to new cells based on training data and/or marker genes. To benchmark Symphony-powered downstream inference against existing classifiers, we

181 followed the same procedure as a benchmarking analysis in Abdelaal et al. (2019) ${ }^{35}$. The benchmark dataset then mapped the test dataset. We used the resulting harmonized feature embedding to predict query cell types using three downstream models: 5-NN, SVM with radial kernel, and multinomial logistic

187 regression. The Symphony-based classifiers achieve consistently high cell type F1-scores (average 188 median F1 of $0.79-0.83$ ) comparable to the top three supervised classifiers for this benchmark

189 (scmapcell, singleCellNet, and SCINA, average median F1 of 0.77-0.83) (Fig. S3a). Notably, as 190 discussed in Abdelaal et al., the median F1-score alone can be misleading given that some classifiers

191 (including SCINA) leave low-confidence cells as "unclassified", whereas we used Symphony to assign a 192 label to every cell. This benchmark is also arguably suboptimal in that the reference in each experiment 193 is comprised of a single dataset (no reference integration involved).

\section{Symphony maps against a large reference within seconds}

195 To demonstrate scalability to large reference atlases, we evaluated Symphony's computational speed.

196 We downsampled a large memory T cell dataset ${ }^{40}$ to create benchmark reference datasets with 20,000 ,

$19750,000,100,000,250,000$, and 500,000 cells (from 12, 30, 58, 156, and 259 donors, respectively).

198 Against each reference, we mapped three different-sized queries: 1,000, 10,000, and 100,000 cells

199 (from 1, 6, and 64 donors) and measured total elapsed runtime (Fig. 3, Table S4). The speed of the 200 reference building process is comparable to that of running de novo integration since they both start 


\section{Total runtime}
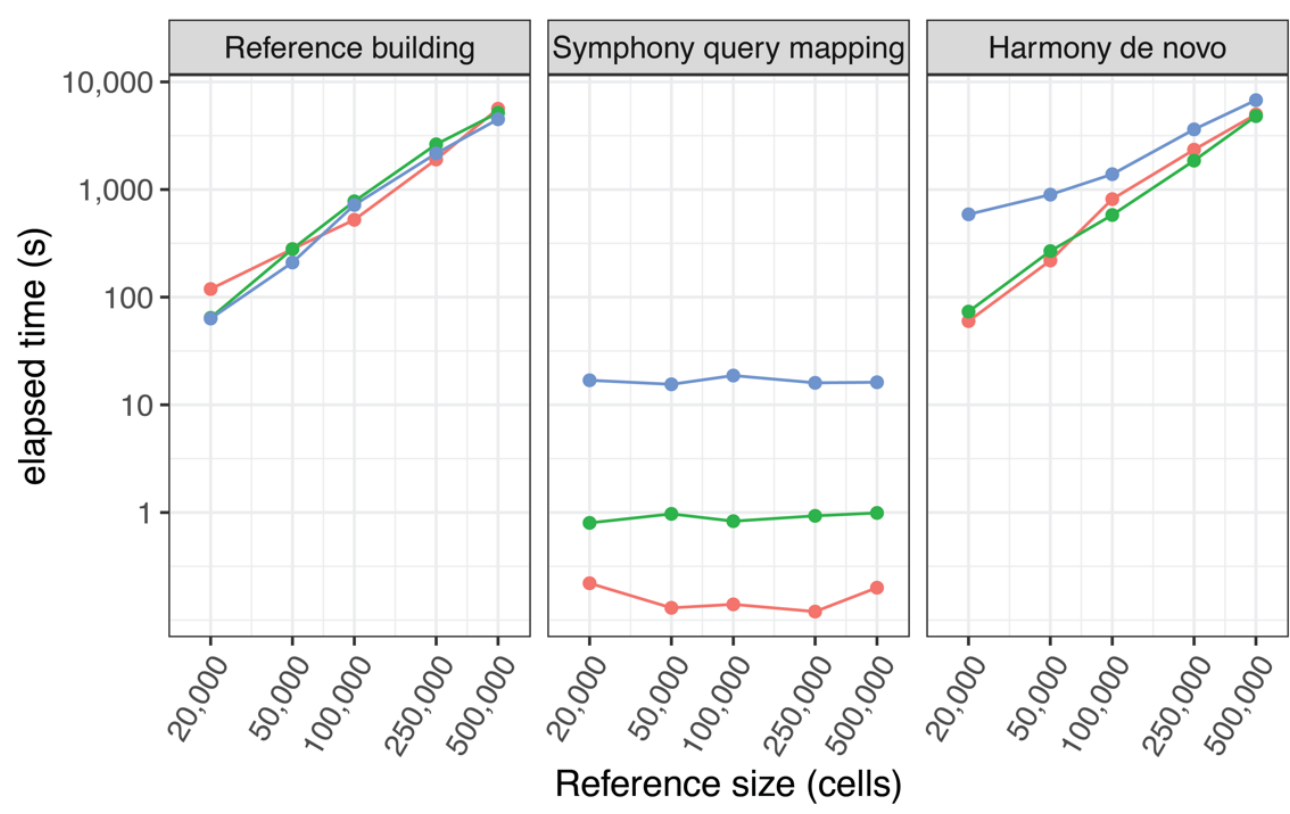

Query size (cells; donors)

$\rightarrow 1,000 ; 1$

$\rightarrow 10,000 ; 6$

$\rightarrow 100,000 ; 64$

Figure 3. Symphony scales mapping to large references within seconds. Total elapsed time (in secs) required to run Symphony reference building starting from gene expression (left), Symphony query mapping starting from query gene expression (middle), or de novo Harmony integration (right) for different-sized reference (x-axis) and query (colors) datasets downsampled from the memory T cell CITE-seq dataset. See Table S4. 
201 with expression data and require a full pipeline of scaling, PCA, and Harmony integration. However, a

202 reference need only be built and saved once in order to map all subsequent query datasets onto it. For 203 instance, initially building a 500,000-cell reference with Symphony took 5,163 seconds (86.1 min) and 204 mapping a subsequent 10,000 -cell query onto it took only 0.99 secs, compared to 4,806 secs (80.1

205 mins) for de novo integration on all cells. Symphony offers a 5000x speedup in this application. These 206 results show that Symphony scales efficiently to map against multimillion-cell references, enabling it to 207 power potential web-based queries within seconds.

reference since the reference cells are modeled post-batch correction (Methods); however, it does

211 cells and batches (Table S4) since the query mapping algorithm solves for the query batch coefficients

212 for each of the reference-defined clusters.

\section{Symphony maps multi-donor, multi-species study to reference of human pancreatic}

\section{4 islet cells}

215 A query dataset might include data from multiple donors, species, and perturbations that create

216 confounding signals obscuring biological signal of interest. Integration algorithms remove these signals

217 in de novo analysis, and it is essential that reference mapping removes them too. Therefore, we

218 designed Symphony to simultaneously handle both tasks: mapping query to reference cells and

219 integration within the query. To test the ability of Symphony to integrate query datasets during mapping,

220 we analyzed reference and query datasets of pancreatic islet cells in which both the reference and

221 query have complex experimental structure (Fig 4a). The reference contained 5,887 pancreatic islet

222 cells from 32 human donors across four independent studies ${ }^{41-44}$, each profiled with a different plate-

223 based scRNA-seq technology (CEL-seq, CEL-seq2, Smart-seq2, and Fluidigm C1). We manually

224 annotated cell types using cluster-specific marker genes within each reference dataset separately

225 (Methods). The query contained 8,569 pancreatic islet cells from 4 human donors and 1,866 cells from

2262 mice, all profiled with inDrop, a droplet-based scRNA-seq technology absent in the reference ${ }^{45}$ (Fig 
a

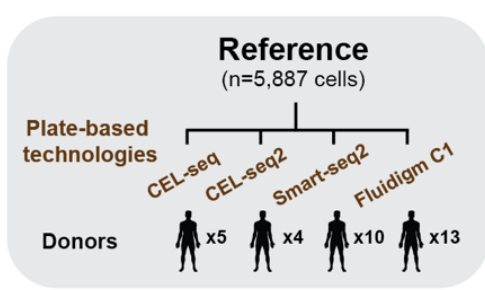

C

Reference cells

Plotted as density
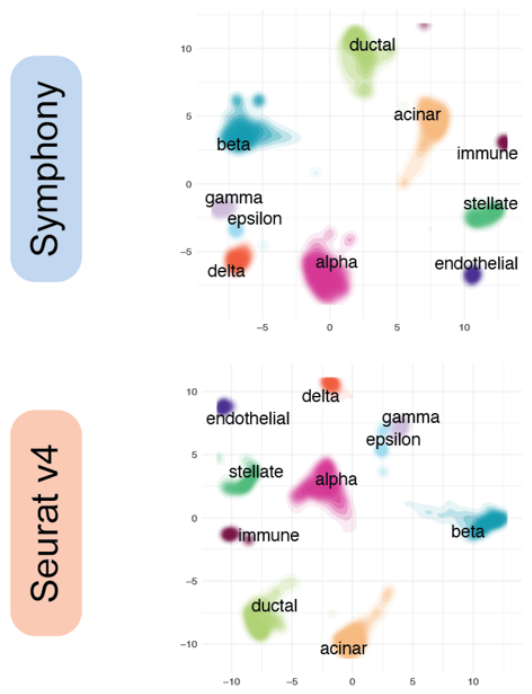

$\mathbf{e}$

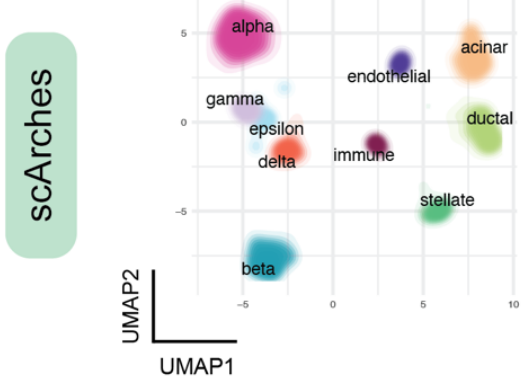

Mixing between reference and query

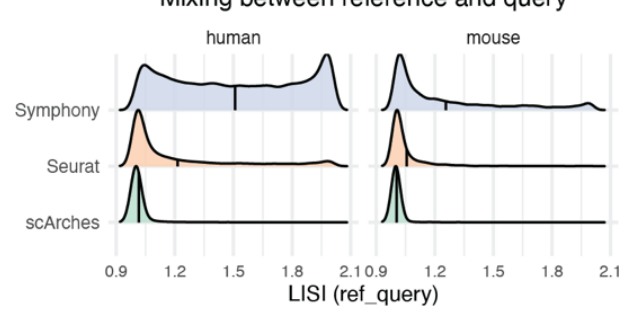

b

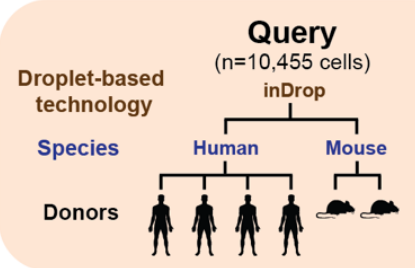

d

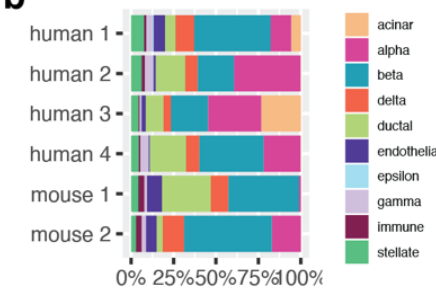

Mapped query cells

Colored by cell type
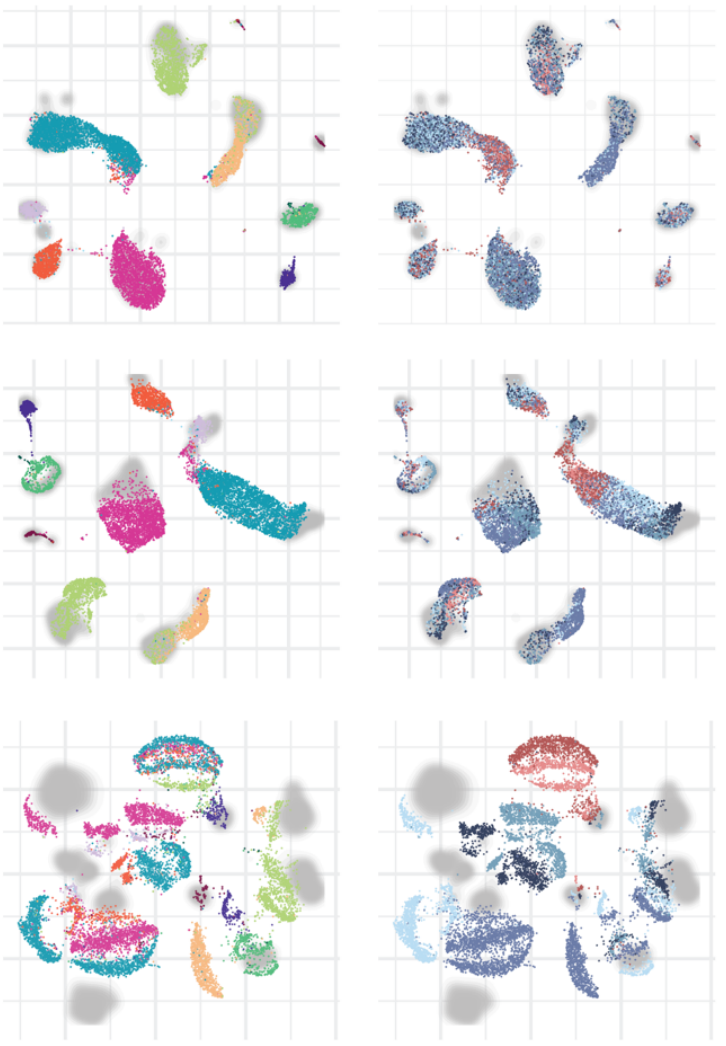

f

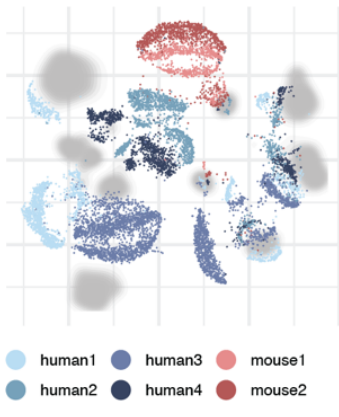

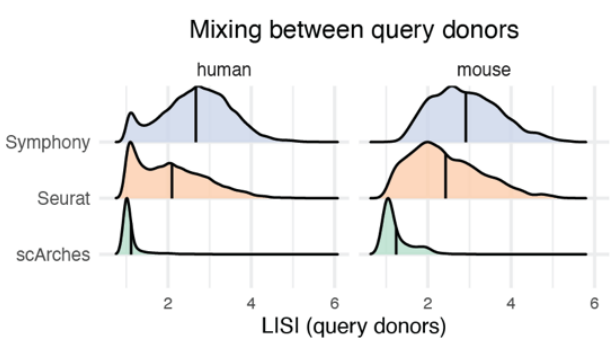

Figure 4. Symphony maps multi-donor, multi-species study to human pancreatic islet cell reference. (a) Schematic of mapping experiment with reference ( $n=5,887$ cells, 32 donors) built from four human pancreas datasets and query dataset ( $n=10,455$ cells, from 4 human donors and 2 mouse donors) sequenced on a new technology (inDrop). (b) Bar plot shows relative proportions of cell types per query donor. We integrated the reference datasets de novo using Harmony, Seurat anchor-based integration, or trVAE, then mapped the query onto the corresponding reference using Symphony, Seurat v4, or scArches, respectively. UMAP plots of resulting joint embeddings showing (c) density of integrated reference cells colored by cell type and (d) query cells colored by cell type as defined by Baron et al. (left) or donor (right) with reference densities plotted in the back in gray. Degree of integration for each method was measured by LISI metric between reference and query labels (e) and LISI between query donors (f) for each query cell neighborhood. Distributions of LISI scores for each method faceted by species and normalized to equal height. See Fig. S4 and S5. 
4b). PCA of the query dataset alone demonstrated the magnitude of the confounding species and donor signals, emphasizing the need for within-query integration (Fig. S4a).

Symphony mapped the multi-species, multi-donor, droplet-based query into the reference by effectively and simultaneously removing the effects of species, donor, and technology (Fig. 4c-d); reference mapping obtained superior integration compared to PCA (mean donor LISI=2.72 compared to 1.45). We predicted that integrating over three nested sources of variation would make it possible to accurately predict query cell types. Using a simple 5-NN classifier in the harmonized embedding, we observed accurate cell-type prediction. Using ground truth labels defined by the original publication ${ }^{45}$, we obtained a median cell type F1-score of 0.96 (overall accuracy $96 \%$ ) for human and median cell type F1 of 0.95 (overall accuracy 91\%) for mouse cells (Fig. S4c-d, Table S5), By mapping against a reference, Symphony is able to overcome strong species effects and simultaneously map analogous cell types between mouse and human.

Next, we evaluated the ability of the other reference mapping algorithms, scArches and Seurat v4, to integrate the same query dataset. For each mapping method, we built a reference using its compatible de novo integration method (Methods, Fig. 4c, S4b). Symphony obtained higher levels of integration than did Seurat and scArches, both between reference and query as well as donors within the query (Fig. 4e-f). Symphony mapping achieves comparable donor mixing to that of Harmony de novo integration of all five datasets (mean mapping LISI=2.67 vs de novo LISI=2.55 in human, 2.91 vs 2.7 in mouse). In contrast, the other mapping methods return less integrated embeddings, when compared to their corresponding de novo methods (mean mapping LISI=2.09 vs de novo LISI=2.83 for

247 Seurat in human, 2.43 vs 2.67 in mouse; 1.12 vs 2.52 for scArches/trVAE in human, and 1.24 vs 3.05 in mouse; Table S6). We then evaluated the accuracy of each mapping with 5-NN cell type classification

(Methods). We observed that Symphony and Seurat performed comparably well, and both 


\section{Localizing query cells along a reference-defined trajectory of human fetal liver}

\section{4 hematopoiesis}

255 A successful mapping method should position cells not only within cell type clusters but also along

256 smooth transcriptional gradients, commonly used to model differentiation and activation processes over

257 time (Fig. 5a). To test Symphony in a gradient mapping context, we built and mapped to a reference

258 atlas profiling human fetal liver hematopoiesis, containing 113,063 liver cells from 14 donors spanning

259 7-17 post-conceptional weeks of age and 27 author-defined cell types, sequenced with 10x 3' chemistry

260 (Fig. 5b, Fig. S6a) ${ }^{46}$. Trajectory analysis of immune populations with the force directed graph (FDG)

261 algorithm ${ }^{46}$ highlights relationships among progenitor and differentiated cell types (Fig. 5c). Notably, the

262 hematopoietic stem cell and multipotent progenitor population branches into three major trajectories,

263 representing the lymphoid, myeloid, and megakaryocyte-erythroid-mast (MEM) lineages. This reference

264 contains two forms of annotation for downstream query inference: discrete cell types and positions

265 along differentiation gradients.

We mapped a query consisting of 21,414 new cells from 5 of the original 14 donors, sequenced

267 with $10 \times 5$ ' chemistry. We first inferred query cell types with k-NN classification (Methods) and

268 confirmed accurate cell type assignment based on the authors' independent query annotations ${ }^{46}$

269 (median cell type F1=0.92 across 14 held-out donor experiments within 3' dataset only, median cell

270 type F1=0.83 for the 5'-to-3' experiment; Fig. S7, Table S7). To evaluate query trajectory inference, we

271 used the Symphony joint embedding to position query cells from the MEM lineage $(n=5,141)$ in the

272 reference-defined trajectory by averaging the 10 nearest reference cell FDG coordinates. The inferred

query trajectory (Fig. 5d) recapitulated known branching from MEM progenitors (MEMPs, brown) into

distinct megakaryocyte (green), erythroid (blue, pink), and mast cell (yellow) lineages. Moreover,

transitions from MEMPs to differentiated types were marked by gradual changes in canonical marker

genes (Fig. 5e): PPBP for megakaryocytes, $H B B$ for erythrocytes, and $K I T$ for mast cells. These

277 gradual expression patterns are consistent with correct placement of query cells along differentiation

278 gradients. 


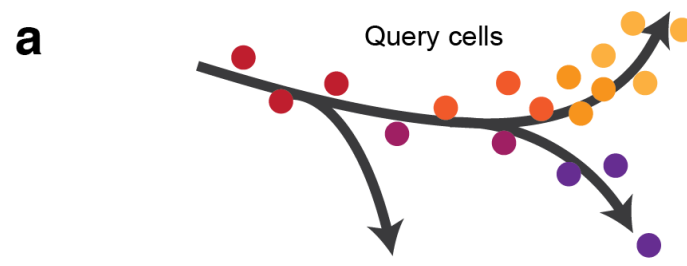

Reference-defined trajectory
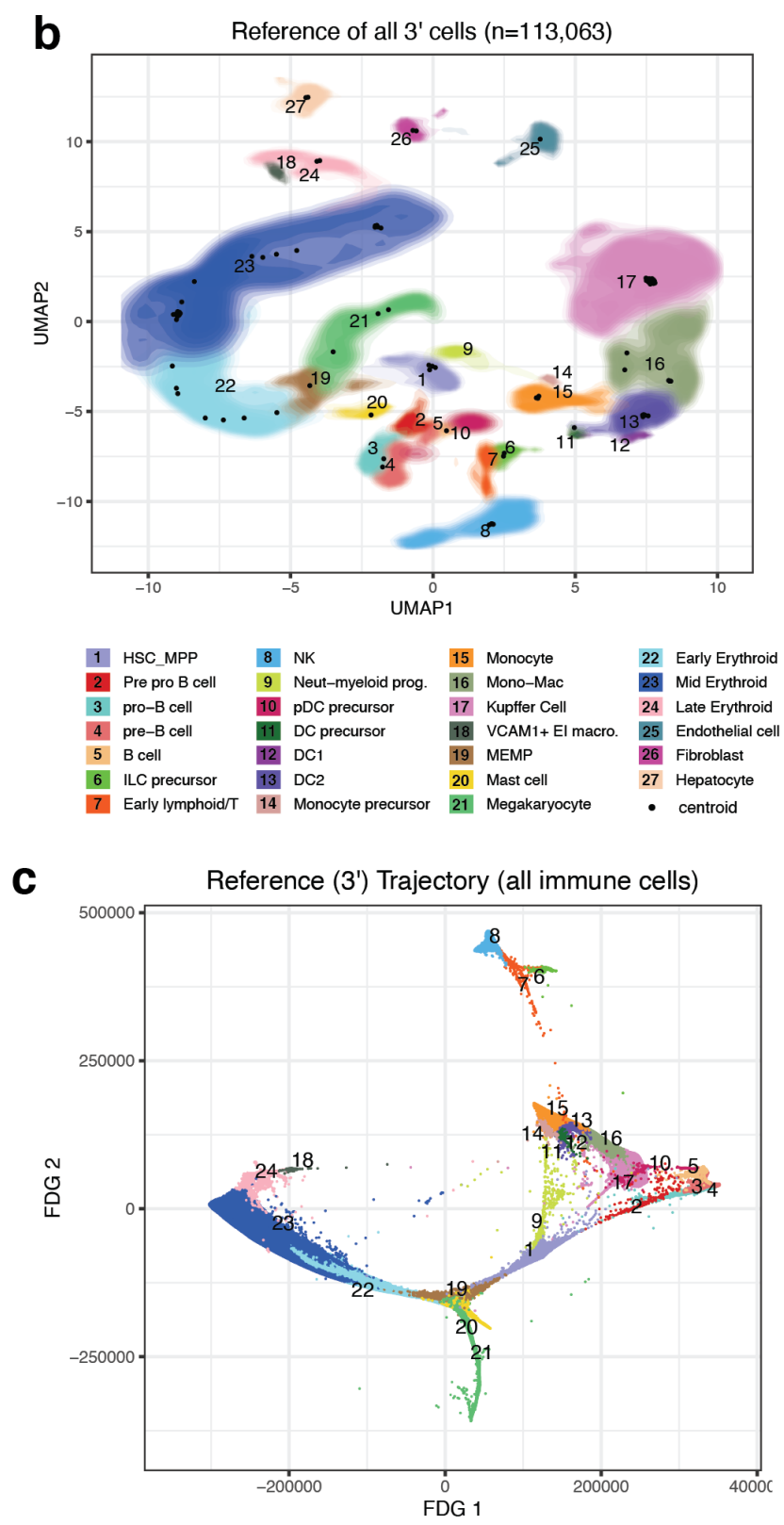
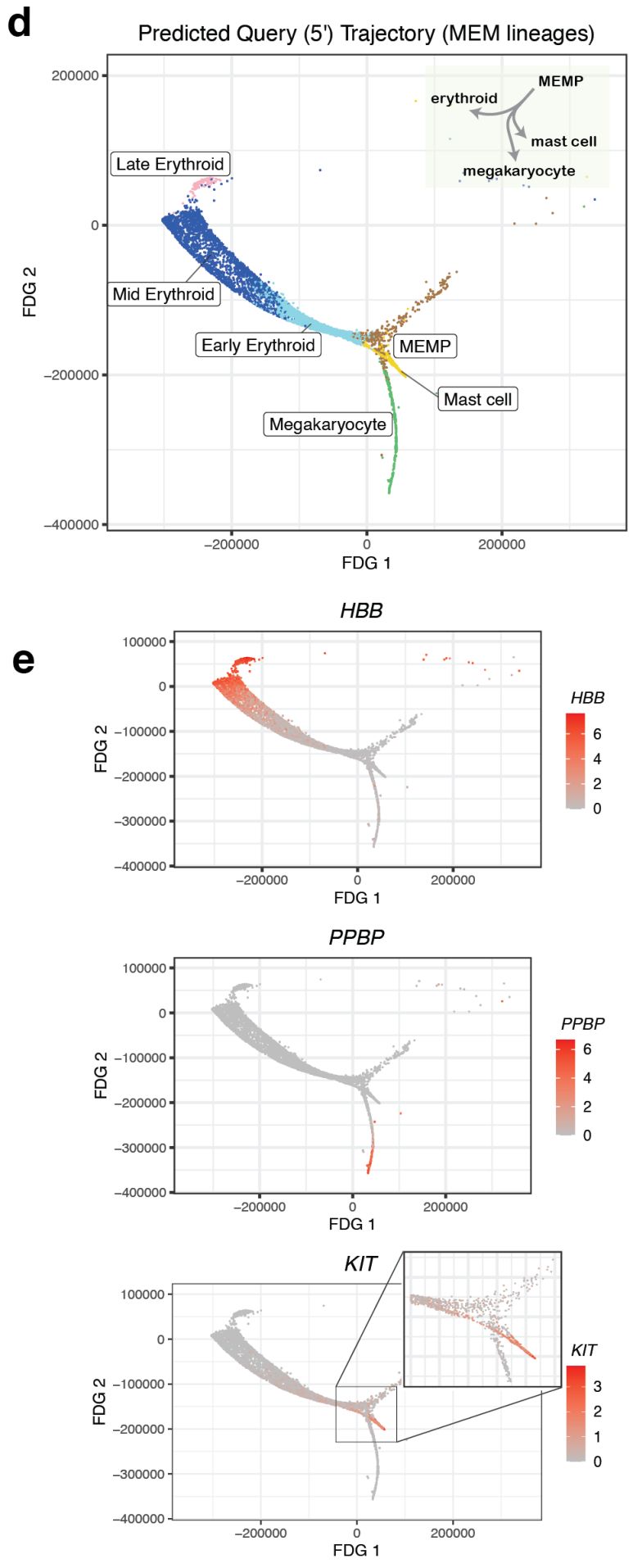

Figure 5. Localizing query cells along a trajectory of fetal liver hematopoiesis. (a) Symphony can precisely place query cells along a reference-defined trajectory. The reference ( $n=113,063$ cells, 14 donors) was sequenced using 10x 3 ' chemistry, and the query ( $n=25,367$ cells, 5 donors) was sequenced with $10 x 5^{\prime}$ chemistry. (b) Symphony reference colored by cell types as defined by Popescu et al. (2019). Contour fill represents density of cells. Black points represent soft-cluster centroids in the Symphony mixture model. (c) Reference developmental trajectory of 3'-sequenced immune cells (FDG coordinates obtained from original authors). Query cells in the MEM lineages ( $n=5,141$ cells) were mapped against the reference and query coordinates along the trajectory were predicted with $10-\mathrm{NN}$ (d). The inferred query trajectory preserves branching within the MEM lineages, placing terminally differentiated states on the ends. (e) Expression of lineage marker genes (PPBP for megakaryocytes, HBB for erythroid cells, and KIT for mast cells). Cells colored by log-normalized expression of gene. See Fig. S6 and S7. 
Inferring query surface protein marker expression by mapping to a reference assayed with CITE-seq

281 Recent technological advances in multimodal single-cell technologies (e.g., CITE-seq) make it possible

282 to simultaneously measure mRNA and surface protein expression from the same cells using

283 oligonucleotide-tagged antibodies ${ }^{47,48}$. With Symphony, we can construct a reference from these data,

284 map query cells from experiments that measure only mRNA expression, and infer surface protein

285 expression for the query cells to expand possible analyses and interpretations (Fig. 6a).

To demonstrate this, we used a CITE-seq dataset that measures the expression of whole-

transcriptome mRNA and 30 surface proteins on 500,089 peripheral blood memory T cells from 271

samples ${ }^{40}$. We leveraged both mRNA and protein features to build a multimodal reference from $80 \%$ of samples ( $n=217)$ and map the remaining $20 \%$ of samples $(n=54)$. Instead of using PCA, which is best for one modality ${ }^{49}$, we used canonical correlation analysis (CCA) to embed reference cells into a space that leverages both. Specifically, CCA constructs a pair of correlated low-dimensional embeddings, one for mRNA and one for protein features, each with a linear projection function akin to gene loadings in PCA. We corrected reference batch effects in CCA space with Harmony and built a Symphony reference (Fig. 6b), saving the gene loadings for the CCA embedding from mRNA features. Then, we mapped the held-out query using only mRNA expression to mimic a unimodal scRNA-seq experiment, reserving the measured query protein expression as a ground truth for validation. We accurately predicted the surface protein expression of each query cell using the 50-NN average from the reference cells in the harmonized embedding. For all proteins, we found strong concordance between predicted and (50-NN smoothed) measured expression (Pearson r: 0.88-0.99, Fig. 6c-d). For all but three proteins, we achieved comparable results with as few as 5 or 10 nearest neighbors (Fig. S8a).

We note that it is also possible to conduct the same analysis with a unimodal PCA-based reference built from the cells' mRNA expression only. This approach has slightly worse performance for some proteins (Pearson r: 0.65-0.97, Fig. S8b-d), demonstrating that a reference built jointly on both mRNA and protein permits better inference of protein expression than an mRNA-only reference, which 
a
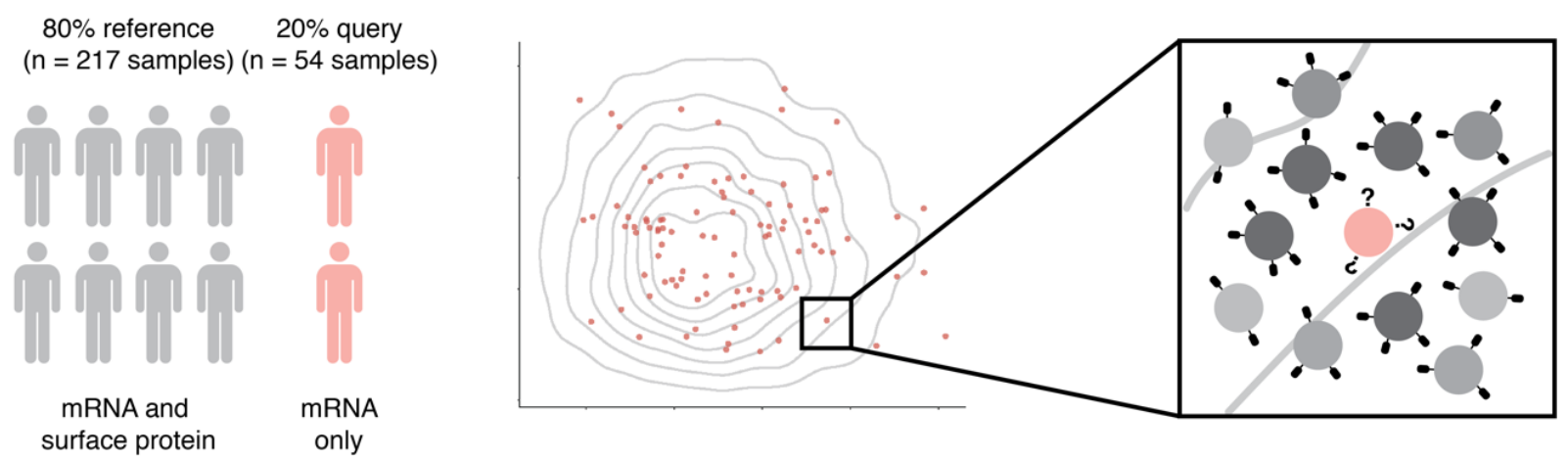

b

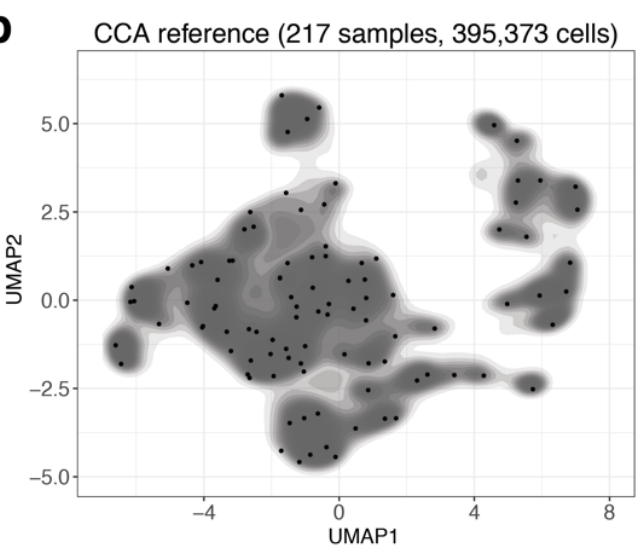

C

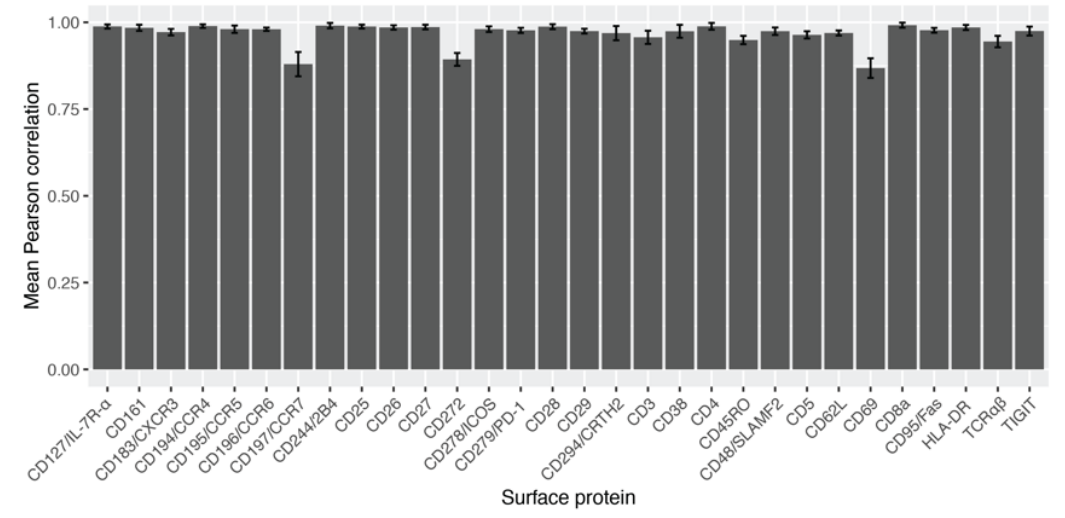

d
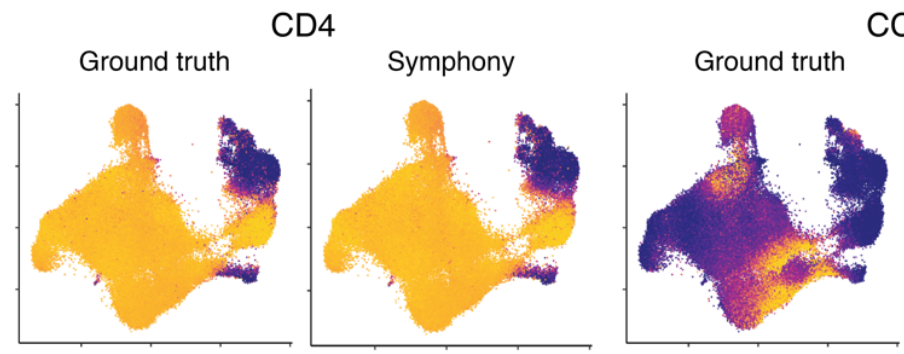

CCR6

Low

Symphony

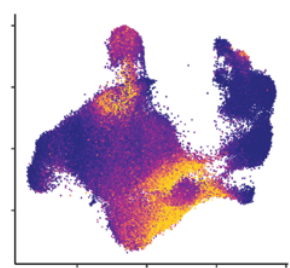

High

Figure 6. Mapping onto a multimodal reference to infer query surface protein expression in memory $\mathrm{T}$ cells. (a) Schematic of multimodal mapping experiment. The dataset was divided into training and test sets $(80 \%$ and $20 \%$ of samples, respectively). The training set was used to build a Symphony reference, and the test set was mapped onto the reference to predict surface protein expression in query cells (pink) based on 50-NN reference cells (gray). (b) Symphony reference built from mRNA/protein CCA embedding. Contour fill represents density of reference cells. Black points represent soft-cluster centroids in the Symphony mixture model. (c) We measured the accuracy of protein expression prediction with the Pearson correlation between predicted and ground truth expression for each surface protein across query cells in each donor. Bar height represents the average per-donor correlation for each protein, and error bars represent standard deviation. (d) Ground truth and predicted expression of CD4, CCR6, and CD69 based on CCA reference. Ground truth is the 50-NN-smoothed expression measured in the CITE-seq experiment. Colors are scaled independently for each marker from minimum (blue) to maximum (yellow) expression. See Fig. S8. 
305 is consistent with previous observations that mRNA expression is not fully representative of protein

306 expression ${ }^{47,48}$. This analysis highlights how users can start with a low-dimensional embedding other

307 than PCA, such as CCA, to better capture rich multimodal information in the reference.

\section{Discussion}

309 Mapping query cells into large, annotated references in real time and without the need to share

310 sensitive information from the reference datasets is becoming increasingly important for reproducible

311 single-cell analysis. We approached this inherently complex, big-data problem using well-established

312 mathematical methods from integration analysis. We framed reference mapping as a specialized case

313 of integration between one relatively small dataset and a second larger, more comprehensive, and

314 previously integrated dataset. Because the reference is already integrated, it is natural to use the same

315 mathematical framework from the integration to perform mapping. For instance, the scArches ${ }^{28}$

316 algorithm uses an autoencoder-based framework to map to references built with autoencoder-based

317 integration algorithms ${ }^{32,33}$. Similarly, Symphony uses the mixture modeling framework to map to

318 references built with Harmony mixture modeling integration. Symphony compresses the reference by

319 extracting relevant reference-derived parameters from the mixture model to map query cells in

320 seconds. With this compression, references can be distributed without the need to share raw

321 expression data or donor-level metadata, which enables data privacy ${ }^{50}$. Symphony compression greatly

322 reduces the size of a reference dataset: for the memory T cell dataset of 500,089 cells, the raw

323 expression matrix is $8.9 \mathrm{~GB}$, whereas the Symphony minimal reference elements are $1.3 \mathrm{MB}$.

Useful reference atlases contain annotations absent in the query, such as cell type labels (Fig.

4), trajectory coordinates (Fig. 5), or multimodal measurements (Fig. 6). Transfer of these annotations from reference to query is an open area of research that includes algorithms for automated cell type classification ${ }^{31,35-38}$. We approach annotation transfer in two steps. We first learn a predictive model in the reference embedding, and then map query cells and use their reference coordinates to predict query annotations. In this two-step approach, Symphony mapping provides a feature space but is otherwise independent from the choice of downstream inference model. In PBMC type prediction (Fig. 
331 S3), we used Symphony embeddings to train multiple competitive classifiers: k-NN, SVM, and logistic

332 regression. In our analyses, we were encouraged to find that a simple k-NN classifier can achieve high

333 performance with only 5-10 neighbors. In practice, users can choose more complex inference models if

334 it is warranted for certain annotation types. Moreover, we expect prediction results to improve with more

335 accurate and reproducible annotation methods, such as consistent cell type taxonomies provided by

336 the Cell Ontology ${ }^{51}$ project and better modeling of multimodal expression data ${ }^{52}$.

Because mapping is a special case of integration, we expected Symphony mapping to

recapitulate the results of de novo Harmony integration. To this end, we defined three conditions under

which Symphony and de novo integration with Harmony yield equivalent results. In subsequent

examples, we showed that Symphony still performs well when the last two conditions are relaxed. The

pancreas query contains more cells than its reference (condition II), while the liver hematopoiesis

reference and query overlap in donors (condition III). Condition I, which requires comprehensive cell

type coverage in the reference, is less flexible. When the query contains a brand new cell type, it will be

aligned to its most transcriptionally similar reference cluster. Note that condition I only pertains to cell

types and not clinical and biological contexts. For instance, we successfully mapped mouse pancreas

query to an entirely human pancreas reference (Fig. 4), because the same pancreatic cell types are

shared in both species. Mapping novel cell types is a current limitation and important direction for future

work. For now, we advise users interested in novel cell type discovery to supplement a Symphony analysis with de novo analyses of the query alone.

Instead of one monolithic reference for all cell types across all tissues and disease, we expect

351 the proliferation of multiple, well-annotated specialized references that focus on fine-grained modeling

352 of diverse biological systems. For instance, the memory T cell reference (Fig. 6) will be useful to

353 annotate fine-grained T cell states, while an unsorted PBMC reference (Fig. 2) would better suit

354 coarse-grained annotation of multiple immune populations. Similarly, a reference with only healthy

355 individuals is useful for annotation of cell types, while a reference with both healthy and diseased

356 individuals is useful for annotation of cell types and pathological cell states. We advise Symphony users 
357 to carefully select the appropriate reference atlas for their study and potentially map to multiple

358 references, as needed. For instance, one may use a PBMC reference to identify and isolate $\mathrm{T}$ cells and 359 a memory $\mathrm{T}$ cell reference to assign fine-grained labels to query $\mathrm{T}$ cells.

As large-scale tissue and whole-organism single-cell reference atlases become available in the

361 near future, Symphony will enable investigators to leverage the rich information in these references to

362 perform integrative analyses and transfer reference coordinates and diverse annotations to new

363 datasets in a rapid and reproducible manner.

\section{Acknowledgements}

365 We thank members of the Raychaudhuri Lab for helpful feedback and comments. We thank members

366 of the Tuberculosis Research Unit (TBRU) LIMAA and Socios En Salud, in particular Megan Murray,

367 Jessica Beynor, Yuriy Baglaenko, Sara Suliman, Ildiko van Rhijn, and Leonid Lecca, for their

368 contributions to generating the memory T cell dataset. We would also like to thank Issac Goh, Muzlifah

369 Haniffa, and other members of the Haniffa Lab for graciously providing preprocessed datasets from

370 their fetal liver hematopoiesis study. This work is supported in part by funding from the National

371 Institutes of Health (1UH2AR067677, U19 Al111224, U01 HG009379, 1R01AR073833, and

372 R01AR063759). The project described was supported by award Number T32GM007753 from the

373 National Institute of General Medical Sciences (JBK). The content is solely the responsibility of the

374 authors and does not necessarily represent the official views of the National Institute of General

375 Medical Sciences or the National Institutes of Health.

\section{Author contributions}

377 I.K., J.B.K., and S.R. conceived the project. J.B.K. and I.K. developed the method and performed the 378 analyses under the guidance of S.R. F.Z. assisted with benchmarking. S.R., A.N., and D.B.M.

379 contributed to generating the memory T cell dataset. A.N. performed analysis of the memory T cell 380 dataset. All authors participated in interpretation and writing the manuscript. 


\section{Declaration of interests}

382 SR receives research support from Biogen.

\section{Figure Legends}

384 Figure 1. Symphony Overview. Symphony comprises two algorithms: Symphony compression (a-b)

385 and Symphony mapping (c-d). (a) To construct a reference atlas, cells from multiple datasets are

386 embedded in a lower-dimensional space (e.g. PCA), in which dataset integration (Harmony) is

387 performed to remove dataset-specific effects. Shape indicates distinct cell types, and color indicates

388 finer-grained cell states. (b) Symphony compression represents the information captured within the

389 harmonized reference in a concise, portable format based on computing summary statistics for the

390 reference-dependent components of the linear mixture model. Symphony returns the minimal reference

391 elements needed to efficiently map new query cells to the reference. (c) Given an unseen query

392 dataset and compressed reference, Symphony mapping precisely localizes the query cells to their

393 appropriate locations within the integrated reference embedding (d). Reference cell locations do not

394 change during mapping. (e) The resulting joint embedding can be used for downstream transfer of

395 reference-defined annotations to the query cells. See Fig. S1.

396 Figure 2. Symphony approximates de novo integration without reintegration of the reference

397 cells. Three PBMC datasets were sequenced with different 10x protocols: 5' (yellow, n=7,697 cells),

398 3'v2 (blue, n=8,380 cells), and 3'v1 (red, n=4,809 cells). We ran Symphony three times, each time

399 mapping one dataset onto a reference built from integrating the other two. (a) Symphony embeddings

400 generated across the three mapping experiments (columns). Top row: cells colored by query (yellow,

401 blue, or red) or reference (gray), with query cells plotted in front. Bottom row: cells colored by cell type:

402 B cell (B), dendritic cell (DC), hematopoietic stem cell (HSC), megakaryocyte (MK), monocyte (Mono),

403 natural killer cell (NK), or T cell (T), with query cells plotted in front. (b) For comparison, gold standard

404 de novo Harmony embedding colored by dataset (top) and cell type (bottom). (c) Distribution of 
405 technology LISI scores for query cell neighborhoods in the Symphony, gold standard, and a standard 406 PCA embeddings on all cells. (d) Distribution of k-NN-corr (Spearman correlation between the 407 similarities between the neighbor-pairs in the Harmony embedding and the similarities between the 408 same neighbor-pairs in the Symphony embedding) across query cells for $k=500$, colored by query 409 dataset. (e) Classification accuracy as measured by cell type F1 scores for query cell type annotation 410 using 5-NN on the Symphony embedding. See Fig. S2.

411 Figure 3. Symphony scales mapping to large references within seconds. Total elapsed time (in

412 secs) required to run Symphony reference building starting from gene expression (left), Symphony 413 query mapping starting from query gene expression (middle), or de novo Harmony integration (right) for 414 different-sized reference (x-axis) and query (colors) datasets downsampled from the memory T cell 415 CITE-seq dataset. See Table S4.

416 Figure 4. Symphony maps multi-donor, multi-species study to human pancreatic islet cell 417 reference. (a) Schematic of mapping experiment with reference ( $n=5,887$ cells, 32 donors) built from 418 four human pancreas datasets and query dataset $(n=10,455$ cells, from 4 human donors and 2 mouse 419 donors) sequenced on a new technology (inDrop). (b) Bar plot shows relative proportions of cell types 420 per query donor. We integrated the reference datasets de novo using Harmony, Seurat anchor-based 421 integration, or trVAE, then mapped the query onto the corresponding reference using Symphony, 422 Seurat v4, or scArches, respectively. UMAP plots of the resulting joint embeddings showing (c) density 423 of integrated reference cells colored by cell type and (d) query cells colored by cell type as defined by 424 Baron et al. (left) or donor identity (right) with reference densities plotted in the back in gray. Degree of 425 integration for each method was measured by LISI metric between reference and query labels (e) and 426 LISI between query donors (f) for each query cell neighborhood. Distributions of LISI scores for each 427 method faceted by species and normalized to equal height. See Fig. S4 and S5.

428 Figure 5. Localizing query cells along a trajectory of fetal liver hematopoiesis. (a) Symphony can 429 precisely place query cells along a reference-defined trajectory. The reference $(n=113,063$ cells, 14 430 donors) was sequenced using $10 \times 3$ ' chemistry, and the query ( $n=25,367$ cells, 5 donors) was 
431 sequenced with $10 \times 5$ ' chemistry. (b) Symphony reference colored by cell types as defined by Popescu 432 et al. (2019). Contour fill represents density of cells. Black points represent soft-cluster centroids in the 433 Symphony mixture model. (c) Reference developmental trajectory of 3'-sequenced immune cells (FDG 434 coordinates obtained from original authors). Query cells in the MEM lineages ( $n=5,141$ cells) were 435 mapped against the reference and query coordinates along the trajectory were predicted with 10-NN 436 (d). The inferred query trajectory preserves branching within the MEM lineages, placing terminally 437 differentiated states on the ends. (e) Expression of lineage marker genes (PPBP for megakaryocytes, 438 HBB for erythroid cells, and KIT for mast cells). Cells colored by log-normalized expression of gene. 439 See Fig. S6 and S7.

Figure 6. Mapping onto a multimodal reference to infer query surface protein expression in

441 memory T cells. (a) Schematic of multimodal mapping experiment. The dataset was divided into 442 training and test sets ( $80 \%$ and $20 \%$ of samples, respectively). The training set was used to build a 443 Symphony reference, and the test set was mapped onto the reference to predict surface protein 444 expression in query cells (pink) based on 50-NN reference cells (gray). (b) Symphony reference built 445 from mRNA/protein CCA embedding. Contour fill represents density of reference cells. Black points 446 represent soft-cluster centroids in the Symphony mixture model. (c) We measured the accuracy of 447 protein expression prediction with the Pearson correlation between predicted and ground truth 448 expression for each surface protein across query cells in each donor. Bar height represents the 449 average per-donor correlation for each protein, and error bars represent standard deviation. (d) Ground 450 truth and predicted expression of CD4, CCR6, and CD69 based on CCA reference. Ground truth is the 451 50-NN-smoothed expression measured in the CITE-seq experiment. Colors are scaled independently 452 for each marker from minimum (blue) to maximum (yellow) expression. See Fig. S8.

\section{Supplementary Figure 1. Overview of reference mapping pipeline and Symphony data}

455 structures. (a) The overall analysis pipeline comprises various functions (orange boxes) that each 456 perform a transformation on the data. Symphony mapping takes in a query gene expression matrix and 
457 a Symphony reference built from integrated reference datasets, and outputs the query cell locations in 458 the harmonized feature embedding. Models trained on the reference feature embedding (e.g. cell type 459 classifier) can transfer annotations to the query for various downstream tasks. (b) Steps of reference 460 building algorithm. Reference datasets spanning multiple batches are aggregated into a single 461 expression matrix on which PCA and Harmony integration is performed. The output of reference 462 compression is the Symphony minimal reference elements, consisting of data structures $\mu, \sigma, U, Y_{\text {cos }}$, $463 N_{r}$, and $C$ (red symbols). $Z_{r_{-} \text {corr }}$ (the harmonized reference embedding) is not used for the mapping 464 calculation but is saved for downstream annotation transfer. (c) Steps of query mapping algorithm, 465 indicating where each reference element is used. Query cells are projected into reference PCA space, 466 clustered to reference centroids, and corrected to harmonized space by removing query batch effects.

467 Supplementary Figure 2. Nearest neighbor correlation (k-NN-corr) metric. The k-NN-correlation 468 metric assesses how well an alternative embedding recapitulates the structure of a gold standard 469 embedding. $\mathrm{k}-\mathrm{NN}$-corr is asymmetric in that it matters which of the two embeddings is selected as the 470 gold standard. Consider a gold standard embedding (a) and two alternative embeddings (b) and (c), 471 representing a good mapping and a bad mapping, respectively. For a given query cell $q$ (red), we 472 identify its top $k$ nearest reference (gray) neighbors in the gold standard embedding $(k=3$ depicted) 473 and calculate the similarity between the query cell and each neighbor. The similarities between the 474 same query-reference neighbor pairs are then calculated in the alternate embedding. k-NN-corr is the 475 Spearman correlation between the similarities in the gold standard vs. alternative embedding, ranging 476 from -1 to +1 . Example k-NN-corr for one query cell and $k=500$ for the (d) Symphony embedding and 477 (e) PCA projection embedding. (f) k-NN-corr distribution across query cells for $\mathrm{k}=500$ and a gold 478 standard Harmony embedding, for either the Symphony embeddings (blue) or a simple PCA projection 479 with no correction step (red), faceted by query dataset.

480 Supplementary Figure 3. Symphony performance against automatic cell type classifiers.

481 Following the cross-technology PBMC benchmarking experiment from Abdelaal et al. (2019), we ran a 482 total of 48 train-test experiments per Symphony-based classifier. Two different versions of the 
483 Symphony feature embeddings were generated depending on variable gene selection method: top

4842000 variable genes (vargenes) or top 20 differentially genes (DEGs) expressed per cell type.

485 Symphony embeddings were used to train 3 downstream classifiers: $k-N N(k=5)$, SVM with radial

486 kernel, and multinomial logistic regression (glmnet) with ridge. (a) Symphony (orange) median cell-type

487 F1 score across 48 train-test experiments compared to supervised methods (green), demonstrating

488 noninferiority to the top supervised methods and stable performance regardless of downstream

489 classification method. Red dot indicates mean of median F1 scores across 48 experiments (used for

490 ordering the methods along the $\mathrm{x}$-axis). (b, c) Median cell type F1 score across 48 experiments for the

491 5-NN classifier with variable gene selection (b) and DEG selection (c). Non-diagonal values represent

492 train on one technology, test on another (42 experiments, all with donor 1). Values along the diagonal

493 indicate train on donor 1 , test on donor 2 of the same technology (6 experiments; missing square

494 because donor 2 not sequenced with 10x v3).

495 Supplementary Figure 4. Comparison of Symphony to alternative reference mapping methods

496 on a cross-species pancreatic islet cell benchmark. (a) Standard PCA pipeline applied to the Baron

497 et al. query dataset exhibits strong species and donor effects, demonstrating the need for within-query

498 integration. We benchmarked Symphony mapping (on a Harmony-integrated reference), Seurat v4

499 mapping (on a Seurat anchor-based-integrated reference), and scArches mapping (on a trVAE-

500 integrated reference). For each approach, we built an integrated reference (b), mapped the query, then

501 predicted query cell types using a 5-NN classifier to transfer annotations using the respective reference

502 embedding. (c) Query cell prediction accuracy by species for each method as measured by cell type F1

503 score, with author-defined ground truth labels. Mouse samples did not have acinar or epsilon cells. The

504 resulting joint cell embedding for each tool was visualized by UMAP (b, d): (b) Reference cells colored

505 by dataset/technology. (d) Query cells colored by correct (green) or incorrect (red) cell type prediction.

506 Supplementary Figure 5. Comparison of de novo integration methods for harmonizing all five

507 pancreatic islet cell datasets. As a comparison to reference mapping (Fig 3), we integrated all five

508 pancreatic islet cell technologies ( $n=16,342$ cells) using 3 de novo integration methods: Harmony, 
509 Seurat anchor-based integration, and trVAE. UMAP visualizations for the integrated embedding colored

510 by batch (a) and cell types (b) for each method. Cell types for reference datasets (c1, celseq, celseq2,

511 smartseq) were defined within each dataset separately based on marker genes. Query cell types were

512 defined by Baron et al. Degree of mixing between reference and query datasets (c) and mixing

513 between query donors (d) was measured with LISI metric on query cell neighborhoods for each

514 method, demonstrating equivalent mixing among de novo integration methods (compare to Fig $3 \mathrm{~d}-\mathrm{e}$ ).

515 Supplementary Figure 6. Mapping to a fetal liver hematopoiesis trajectory. (a) Size and cell type

516 composition of each donor sample in the 10x 3' dataset across 27 author-defined cell types from

517 Popescu et al. (2019). pcw = post-conception weeks. (b) Library complexity for each sample in 10x 3'

518 and 10x 5' datasets, showing low complexity for donor F2 and F5 5'-sequenced samples (removed

519 from further analysis). (c) UMAP projections of query cells into reference UMAP space after Symphony

520 mapping, faceted by query donor, colored by cell type. Reference UMAP embedding in bottom-right.

\section{Supplementary Figure 7. Fetal liver hematopoiesis cell type classification confusion matrices.}

522 We performed two versions of the reference mapping experiments to assess cell type classification

523 accuracy across 27 fine-grained cell types: (1) using exclusively 10x 3' data, we mapped one held-out

524 donor against a reference constructed from the remaining 13 donors (total 14 mapping experiments),

525 (2) mapping all $10 \times 5$ ' cells against all 10x 3' cells. Cell type confusion matrices are shown for a 30-NN

526 cell type classifier (a) aggregated across the 14 held-out donor experiments using exclusively 3' data

527 and (b) the 5'-to-3' experiment mapping the full 5' query ( $n=21,414, n=5$ donors) against the full 3'

528 reference ( $n=113,063$ cells, 14 donors), colored by the proportion of the true cell type that was

529 classified correctly. True cell type is defined by the original authors (Popescu et al., 2019). Rows (true

530 query cell types) are sorted by hierarchical clustering on the average gene expression (all genes) for

531 the cell types to order similar types together. Bar graph (right) shows population size for each cell type.

532 Supplementary Figure 8. Inferring query surface protein expression in memory T cells. (a) Mean

533 Pearson correlation for CCA reference between k-NN predicted protein expression and ground truth for

534 different values of $k$. (b) Symphony reference built from a standard mRNA PCA embedding (reference 
535 protein values were not used to build embedding but treated as annotations only). Contour fill

536 represents density of reference cells. Black points represent soft-cluster centroids in the Symphony

537 mixture model. (c) We measured the accuracy of protein expression prediction based on the PCA

538 reference with the Pearson correlation between predicted and ground truth expression for each surface

539 protein across query cells in each donor. Bar height represents the average per-donor correlation for

540 each protein, and error bars represent standard deviation. (d) Ground truth and predicted expression of

541 CD4, CCR6, and CD69 based on PCA reference. Ground truth is the 50-NN-smoothed expression

542 measured in the CITE-seq experiment. Colors are scaled independently for each marker from minimum

543 (blue) to maximum (yellow) expression.

545 Supplementary Table 1. Links to datasets used in the study.

546 Supplementary Table 2. Canonical lineage markers (Wilcoxon rank sum test and auROC statistic) and

547 top 10 differentially expressed genes per cluster used to assign cell types in 10x PBMCs.

548 Supplementary Table 3. Cell type classification confusion matrices for the three 10x PBMCs mapping 549 experiments.

550 Supplementary Table 4. Runtime scalability analysis results (downsampling memory T cell dataset), 551 showing effect of reference and query size, number of query cells or donors, and number of reference 552 centroids or embedding dimensions on elapsed time (in secs).

553 Supplementary Table 5. Cell type classification confusion matrix for multi-donor, multi-species 554 pancreatic islet cell benchmarking example (mapping Baron et al. 2016 as query) among the reference 555 mapping methods evaluated.

556 Supplementary Table 6. Degree of mixing between reference and query cells (ref_query LISI) and 557 between donors within the query (query donor LISI) as well as runtime comparison across different 558 reference mapping methods and corresponding de novo integration methods (Symphony/Harmony, 559 Seurat v4/Seurat, and trVAE/scArches) for multi-donor, multi-species pancreas benchmarking example. 
560 Supplementary Table 7. Cell type classification confusion matrix for mapping 10x 5'-sequenced fetal

561 liver cells onto an atlas of 3'-sequenced fetal liver cells (Popescu et al. 2019). True labels provided by

562 the original authors, and predictions were made using a 30-NN classifier. 


\section{Methods}

564 1. Symphony

565

566

567

568

569

570

571

572

573

574

575

576

577

578

579

580

581

582

583

584

585

586

\subsection{Symphony overview}

The goal of single-cell reference mapping is to embed newly assayed query cells into an existing comprehensive reference atlas, facilitating the automated transfer of annotations from the reference to the query. The optimal mapping method needs to be able to operate at various levels of resolution, capture continuous intermediate cell states, and scale to multimillion cells ${ }^{27}$. Consider a scenario in which we wish to map a query of $m$ cells against reference datasets with $n$ cells, where $m<<n$. Unsupervised integration of measurements across donors, studies, and technological platforms is the standard way to compare single cell datasets and identify cell types. Hence, a "gold standard" reference mapping strategy might be to run Harmony integration on all $m+n$ cells de novo. However, this approach is impractical because it is cumbersome and time-intensive to process all the cell-level data for the reference datasets every time a user wishes to reharmonize it with a query. Instead, we envision a pipeline where a reference atlas need only be carefully constructed and integrated once, and all subsequent queries can be rapidly mapped into the same stable reference embedding.

Symphony is a reference mapping method that efficiently places query cells in their precise location within an integrated low-dimensional embedding of reference cells, approximating de novo harmonization without the need to reintegrate the reference cells. Symphony is comprised of two algorithms: reference compression and mapping. Expanding upon the linear mixture model framework introduced in Harmony ${ }^{17}$, Symphony compression takes in an integrated reference and faithfully compresses it by capturing the components of the model into efficient data structures. The output of reference compression is the minimal set of elements needed for mapping (Fig. S1b). The Symphony mapping algorithm takes as input a new query dataset as well as minimal reference elements and returns the appropriate locations of the query cells within the integrated embedding (Fig. S1c). 
587 Once a harmonized reference is constructed and compressed using Symphony, subsequent mapping

588 of query cells executes within seconds (Fig. 3). Efficient implementations of Symphony are available as

589 part of an R package at https://github.com/immunogenomics/symphony, along with several

590 precomputed references constructed from public scRNA-seq datasets. The following sections introduce

591 the Symphony model, then describes Symphony compression and mapping in terms of the underlying

592 data structures and algorithms. We also provide Supplementary Equations containing more detailed

593 derivations for reference compression terms.

594 Glossary

595 We define all symbols for data structures used in the discussion of Symphony below, including their 596 dimensions and possible values. Dimensions are in terms of the following parameters:

$597 \quad n$ : the number of reference cells

$598 \quad-m$ : the number of query cells

$599 \quad-N$ : the total number of cells $(n+m)$

$600 \quad-g$ : the number of genes in the reference after any gene selection

$601 \quad d$ : the dimensionality of the embedding (e.g. PCs). $d$ applies to both reference and query.

$602 \quad b$ : the number of batches in the reference

603 - c: the number of batches in the query

$604 \quad-k$ : the number of clusters in the mixture model for reference integration (representing latent cell $605 \quad$ states)

\section{Reference-related symbols:}
$G_{r} \in \mathbb{R}^{g \times n}$
Input reference gene expression matrix, prior to scaling.
$G_{r s} \in \mathbb{R}^{g \times n}$
Scaled reference gene expression matrix.
$X_{r} \in\{0,1\}^{b \times n}$
One-hot design matrix assigning reference cells (columns) to batches (rows).
$X_{r}^{\prime} \in\{0\}^{c \times n}$
Zero matrix assigning reference cells (columns) to query batches (rows).
All values are 0 because reference cells do not belong to query batches.
This term is used in the derivation for the reference compression terms. 


$$
\begin{array}{ll}
\mu \in \mathbb{R}^{g \times 1} & \text { Reference gene means used to center each gene for PCA. } \\
\sigma \in \mathbb{R}^{g \times 1} & \text { Reference gene standard deviations used to scale each gene for PCA. } \\
U \in \mathbb{R}^{g \times d} & \text { Gene loadings from the original PCA (before Harmony integration). } \\
Z_{r} \in \mathbb{R}^{d \times n} & \text { Original (non-harmonized) PC embedding for reference cells. } \\
\hat{Z}_{r} \in \mathbb{R}^{d \times n} & \text { Integrated embedding for reference cells in harmonized PC (hPC) space, } \\
& \text { as output by Harmony. } \\
R_{r} \in[0,1]^{k \times n} & \text { Soft cluster assignment of reference cells (columns) to clusters (rows), as } \\
& \text { output by Harmony. Each column is a probability distribution that sums to } \\
Y_{\cos } \in \mathbb{R}^{d \times k} & \text { Cluster centroid locations in the harmonized embedding, L2 normalized. } \\
B_{r} \in \mathbb{R}^{k \times(1+b) \times d} & \text { 3D tensor of the estimated parameters (betas and intercepts) of the linear } \\
N_{r} \in \mathbb{R}^{k \times 1} & \text { mixture model for each of } k \text { clusters for the reference cells. } \\
& \text { First reference compression term. Vector containing the size of each of } \\
& \text { the } k \text { clusters, effectively the number of reference cells contained within } \\
\text { them. } & \text { Second reference compression term. } \\
C \in \mathbb{R}^{k \times d} & \text { Symphony minimal reference elements comprising } \mu, \sigma, U, Y_{\text {cos }}, N_{r}, C .
\end{array}
$$

\section{Query-related symbols:}

$$
\begin{aligned}
& G_{q} \in \mathbb{R}^{g \times m} \quad \text { Input query gene expression matrix, prior to scaling. } \\
& G_{q s} \in \mathbb{R}^{g \times m} \quad \text { Query gene expression matrix, scaled by reference gene means } \mu \text { and } \\
& \text { standard deviations } \sigma \text {. } \\
& X_{q} \in\{0,1\}^{c \times m} \quad \text { Design matrix assigning query cells (columns) to query batches (rows). } \\
& Z_{q} \in \mathbb{R}^{d \times m} \quad \text { Query cell locations in original (non-harmonized) PC embedding. } \\
& \hat{Z}_{q} \in \mathbb{R}^{d \times m} \quad \text { Approximate query cell locations in integrated embedding (hPC space). Output } \\
& \text { of Symphony reference mapping. } \\
& R_{q} \in[0,1]^{k \times m} \quad \text { Soft cluster assignment of query cells (columns) to clusters (rows). Each } \\
& \text { column is a probability distribution that sums to } 1 \text {. } \\
& B_{q} \in \mathbb{R}^{k \times(1+c) \times d} \quad 3 \mathrm{D} \text { tensor of the estimated parameters (betas and intercepts) of the linear } \\
& \text { mixture model for each of } k \text { clusters. }
\end{aligned}
$$


609 Symphony and Harmony both use a linear mixture model framework, but the two methods perform

610 different tasks: Harmony integrates a reference, whereas Symphony compresses the reference and

611 enables efficient query mapping. To motivate the Symphony model, it is helpful to first briefly review the

612 mixture model, which serves as the basis. Harmony integrates scRNA-seq datasets across batches

613 (e.g. multiple donors, technologies, studies) and projects the cells into a harmonized embedding where

614 cells cluster by cell type rather than batch-specific effects. Harmony takes as input a low-dimensional

615 embedding of cells $(Z)$ and design matrix with assignments to batches $(X)$ and outputs a harmonized

616 embedding $(\hat{Z})$ with batch effects removed. Briefly, Harmony works by iterating between two

617 subroutines-maximum diversity clustering and linear mixture model correction-until convergence. In

618 the clustering step, cells are probabilistically assigned to soft clusters with a variant of soft $k$-means with

619 a diversity penalty favoring clusters represented by multiple datasets rather than single datasets. In the

620 correction step, each cluster learns a cluster-specific linear model that explains cell locations in PC

621 space as a function of a cluster-specific intercept and batch membership. Then, cells are corrected by

622 cell-specific linear factors weighted by cluster membership to remove batch-dependent effects. The full

623 algorithm and implementation are detailed in Korsunsky et al. (2019) ${ }^{17}$.

624 In the scenario of mapping $m$ query cells against $n$ reference cells, the de novo integration strategy

625 would model all cells as in (1), where the $H$ subscript denotes the Harmony solution, in contrast to the

626 Symphony model which is presented in (2). Let $X_{H} \in\{0,1\}^{(c+b) \times(m+n)}$ represent the one-hot encoded

627 design matrix assigning all cells across batches. $X_{H}^{*}$ denotes $X_{H}$ augmented with a row of $1 \mathrm{~s}$ for the

628 batch-independent intercept term: $X_{H}^{*}=1|| X_{H}$. The intercept terms represent cluster centroids (location

629 of "experts" in the mixture of experts model). $Z_{H}$ represents the low-dimensional PCA embedding of all

630 cells. $R_{H}$ represents the probabilistic assignment of cells across $k$ clusters, and $\operatorname{diag}\left(R_{H k}\right) \in \mathbb{R}^{N \times N}$

631 denotes the diagonalized $k$ th row of $R_{H}$. For each cluster $k$, the parameters of the linear mixture model

$632 B_{k} \in \mathbb{R}^{(1+c+b) \times d}$ can therefore be solved for as in (1), using ridge regression with ridge penalty

633 hyperparameter $\lambda$. Note that we do not penalize the batch-independent intercept term: $\lambda_{0}=0$,

$634 \quad \forall_{a \in[1:(c+b)]} \lambda_{a}=1$ 
De novo Harmony model:

$$
B_{k}=\left(X_{H}^{*} \operatorname{diag}\left(R_{H k}\right) X_{H}^{* T}+\lambda I\right)^{-1} X_{H}^{*} \operatorname{diag}\left(R_{H k}\right) Z_{H}^{T}
$$

636 The goal of Symphony mapping is to add new query cells to the model in order to estimate and remove

637 the query batch effects. Symphony mapping approximates de novo Harmony integration on all cells,

638 except the reference cell positions in the harmonized embedding do not change. In order for Symphony 639 mapping to be equivalent to de novo Harmony, several conditions must be met:

I. All cell states represented in the query dataset are captured by the reference datasets-i.e.

We consider these to be fair assumptions for large-scale reference atlases, allowing Symphony to make three key approximations:

II. The number of reference cells is much larger than the query $(m<<n)$.

III. The query dataset is obtained independent of the reference datasets-i.e. the reference batch design matrix $\left(X_{r}\right)$ has no interaction with the query batch design matrix $\left(X_{q}\right)$.

Given these approximations, we can thereby harmonize the reference cells a priori and save the reference-dependent portions of the Harmony mixture model (Supplementary Equations). In

Symphony, we model the reference cells as already harmonized with batch effects removed, so we can

657 thereafter ignore the reference design matrix structure. The Symphony design matrix $X \in[0,1]^{c \times N}$

658 assigns all cells (reference and query) to query batches only. $X^{*}$ denotes $X$ augmented with a row of $1 \mathrm{~s}$ 
$659\left(X_{[0,]}^{*}\right)$ corresponding to the batch-independent intercepts (we model the intercepts for all cells). The 660 remaining $c$ rows $\left(X_{[1: c,]}^{*}\right)$ represent the one-hot batch assignment of the cells among the $c$ query

661 batches. Note that for the reference cell columns, these values are all 0 since the reference cells do not 662 belong to any query batches. The parameters $\left(B_{q k} \in \mathbb{R}^{(1+c) \times d}\right)$ of the model for each cluster $k$ can 663 then be solved for as in (2). Similar to Harmony, we use ridge regression penalizing the non-intercept terms, where $\lambda_{0}=0, \forall_{a \in[1: c]} \lambda_{a}=1$.

\section{Symphony model:}

$$
B_{q k} \approx\left(X^{*} \operatorname{diag}\left(R_{k}\right) X^{* T}+\lambda I\right)^{-1} X^{*} \operatorname{diag}\left(R_{k}\right) Z^{T}
$$

666 The matrix $R \in \mathbb{R}^{k \times N}$ denotes the assignment of query and reference cells (columns) across the 667 reference clusters (rows). $Z \in \mathbb{R}^{d \times N}$ denotes the horizontal matrix concatenation of the uncorrected 668 query cells in original PC space $\left(Z_{q}\right)$ and corrected reference cells in harmonized space $\left(\hat{Z}_{r}\right)$. For each cluster $k$, let matrix $B_{q k} \in \mathbb{R}^{(1+c) \times d}$ represent the query parameters to be estimated. The first row of $B_{q k}$ represents the batch-independent intercept terms, and the remaining $c$ rows of $B_{q k}$ represent the query batch-dependent coefficients, which can be regressed out to harmonize the query cells with the reference. Note that the intercept terms from Symphony mapping should equal the cluster centroid locations from the integrated reference since the harmonized reference cells are modeled only by a weighted average of the centroid locations for the clusters over which it belongs (and a cell-specific residual). Hence, the reference cell positions should not change when removing query batch effects.

The matrices $X^{*}, R_{k}$, and $Z$ in (2) can be partitioned into query and reference-dependent portions. In the Supplementary Equations, we show in detail how the reference-dependent portions can be further simplified into a $k \times 1$ vector and $k \times d$ matrix $\left(N_{r}\right.$ and $\left.\mathrm{C}\right)$, which we call "reference compression terms." Intuitively, the vector $N_{r}$ contains the size (in cells) of each reference cluster. The matrix $C=R_{r} \hat{Z}_{r}^{T}$ does not have as intuitive an explanation but follows from the derivation (Supplementary Equations). These terms can be computed at the time of reference building and saved as part of the minimal reference elements to reduce the necessary computations during mapping. 


\section{3}

684

\subsection{Reference building and compression}

Reference compression is the key idea that allows for the efficient mapping of new query cells onto the harmonized reference embedding without the need to reintegrate all cells. To construct a Symphony reference with minimal elements needed for mapping, reference cells are first harmonized in a lowdimensional space (e.g. PCs) to remove batch-dependent effects. Symphony then compresses the Harmony mixture model components to be saved for subsequent query mapping.

\section{Data structures}

Symphony takes as input a gene expression matrix for reference cells $\left(G_{r}\right)$ and corresponding one-hotencoded design matrix $\left(X_{r}\right)$ containing metadata about assignment of cells to batches. It outputs a set of data structures, referred to as the Symphony minimal reference elements, that captures key information about the reference embedding that can be subsequently used to efficiently map previously unseen query cells (Algorithm 1). These components include the gene mean $(\mu)$ and standard deviation $(\sigma)$ used to scale the genes, the PCA gene loadings $(U)$, the final L2-normalized cluster centroid locations $\left(Y_{\text {cos }}\right)$, and precomputed values which we call the "reference compression terms" $\left(N_{r}\right.$ and $C$ ) that expedite the correction step of query mapping (Supplementary Equations). These elements are a subset of the components available once Harmony integration is applied to the reference cells. Note that other input embeddings, such as canonical correlation analysis (CCA), may be used in place of PCA as long as the gene loadings to perform query projection into those coordinates are saved.

Table 1 lists the Symphony minimal reference elements required to perform mapping. Table 2 shows additional components of a "full" Harmony reference that are not included in the Symphony reference elements. Importantly, the dimensions of the Symphony data structures do not require information on the $n$ individual reference cells and hence do not scale with the raw number of reference cells. Rather the components scale with the biological complexity captured (i.e. number of clusters $k$ and dimensionality of embedding d). Conversely, the Harmony data structures store information on a percell basis $(n)$. Note that in practice the integrated embedding of reference cells $\left(\hat{Z}_{r}\right)$ listed in Table 2 is 
needed to perform downstream transfer of annotations from reference to query cells (e.g. k-NN), but it is not required during any computations of the mapping step.

\section{Table 1: Symphony minimal reference elements}

\begin{tabular}{|c|l|}
\hline$\mu \in \mathbb{R}^{g \times 1}$ & Reference gene means used to center each gene for PCA. \\
\hline$\sigma \in \mathbb{R}^{g \times 1}$ & Reference gene standard deviations used to scale each gene for PCA. \\
\hline$U \in \mathbb{R}^{g \times d}$ & Gene loadings to project from expression to PCA (or CCA) space \\
\hline$Y_{\text {cos }} \in \mathbb{R}^{d \times k}$ & Cluster centroid locations in harmonized PC space, L2 normalized. \\
\hline$N_{r} \in \mathbb{R}^{k \times 1}$ & $\begin{array}{l}\text { First reference compression term. Vector containing the size of each of the } k \\
\text { clusters, effectively the number of reference cells contained within them. }\end{array}$ \\
\hline$C \in \mathbb{R}^{k \times d}$ & Second reference compression term. \\
\hline
\end{tabular}

713 Table 2: Additional components of Harmony reference

\begin{tabular}{|c|l|}
\hline$G_{r} \in \mathbb{R}^{g \times n}$ & Input reference gene expression matrix, prior to scaling. \\
\hline$X_{r} \in\{0,1\}^{b \times n}$ & Design matrix assigning reference cells (columns) to reference batches (rows). \\
\hline$B_{r} \in \mathbb{R}^{k \times(1+b) \times d}$ & $\begin{array}{l}\text { 3D tensor of the estimated parameters (betas and intercepts) of the linear } \\
\text { mixture model for each of } k \text { clusters for the reference cells. }\end{array}$ \\
\hline$\hat{Z}_{r} \in \mathbb{R}^{d \times n}$ & $\begin{array}{l}\text { Integrated embedding for reference cells in harmonized PC ("hPC") space, as } \\
\text { output by Harmony. }\end{array}$ \\
\hline$R_{r} \in[0,1]^{k \times n}$ & $\begin{array}{l}\text { Soft cluster assignment of reference cells (columns) to clusters (rows), as output } \\
\text { by Harmony. Each column is a probability distribution that sums to 1. }\end{array}$ \\
\hline
\end{tabular}

\section{Algorithm}

716 Starting from reference cell gene expression, we first perform within-cell library size normalization (if not

717 already done) and variable gene selection to obtain $G_{r}$, scaling of the genes to have mean 0 and

718 variance 1 (saving $\mu$ and $\sigma$ for each gene), and PCA to embed the reference cells in a low-dimensional

719 space, saving the gene loadings $(U)$ (Implementation Details). Then, the PCA embedding $\left(Z_{r}\right)$ and

720 batch design matrix $\left(X_{r}\right)$ are used as input to Harmony integration to harmonize over batch-dependent

721 sources of variation. Given the resulting harmonized embedding $\left(\hat{Z}_{r}\right)$ and final soft assignment of 
722 reference cells to clusters $\left(R_{r}\right)$, the locations of the final reference cluster centroids $Y \in \mathbb{R}^{d \times k}$ can be

723 calculated as in (3) and saved.

$$
Y=\hat{Z}_{r} R_{r}^{T}
$$

724 Symphony then computes the reference compression terms $N_{r}$ (intuitively, the number of cells per

725 cluster) and $C$, which does not have an intuitive explanation but can be directly computed as $C=R_{r} \hat{Z}_{r}^{T}$.

726 Refer to the Supplementary Equations for a complete mathematical derivation of the compression

727 terms. Symphony reference building ultimately returns the minimal reference elements: $\mu, \sigma, U, Y_{c o s}, N_{r}$, 728 and $C$ (Fig. S1a).

\subsection{Symphony mapping}

742 The Symphony mapping algorithm localizes new query cells to their appropriate locations in the

743 harmonized embedding without the need to run integration on the reference and query cells altogether.

744 The joint embedding of reference and query cells can be used for downstream analyses, such as

745 transferring cell type annotations from the reference cells to the query cells. 


\section{Data structures}

747 Symphony mapping takes as input the gene expression matrix for query cells $\left(G_{q}\right)$, query design matrix 748 assigning query cells to batches $\left(X_{q}\right)$, and the precomputed minimal elements for a reference $(\operatorname{Ref})$. It 749 outputs a query object containing the locations of query cells in the integrated reference embedding $750\left(\hat{Z}_{q} ;\right.$ Algorithm 2). Table 3 lists the components of the query object that is returned by Symphony.

751 Table 3: Components of Symphony query

\begin{tabular}{|c|l|}
\hline$G_{q} \in \mathbb{R}^{g \times m}$ & Input query gene expression matrix, prior to scaling. \\
\hline$X_{q} \in\{0,1\}^{c \times m}$ & Design matrix assigning query cells (columns) to query batches (rows). \\
\hline$Z_{q} \in \mathbb{R}^{d \times m}$ & Query cell locations in original (non-harmonized) PC embedding. \\
\hline$\hat{Z}_{q} \in \mathbb{R}^{d \times m}$ & Approximate query cell locations in integrated embedding (hPC space). \\
\hline$R_{q} \in[0,1]^{k \times m}$ & $\begin{array}{l}\text { Soft cluster assignment of query cells (columns) to clusters (rows). Each } \\
\text { column is a probability distribution that sums to 1. }\end{array}$ \\
\hline$B_{q} \in \mathbb{R}^{k \times(1+c) \times d}$ & $\begin{array}{l}3 \mathrm{D} \text { tensor of the estimated parameters (betas and intercepts) of the linear } \\
\text { mixture model for each of } k \text { clusters. }\end{array}$ \\
\hline
\end{tabular}

\section{Algorithm}

754 The input to the query mapping procedure is a gene expression matrix $\left(G_{q}\right)$ and design matrix $\left(X_{q}\right)$ for 755 query cells, and the output is the locations of the cells in the harmonized embedding $\left(\hat{Z}_{q}\right)$. At a high 756 level, the mapping algorithm first projects the query cells into the original, non-harmonized PC space as 757 the reference cells using the reference gene loadings $(U)$ and assigns probabilistic cluster membership 758 across the reference cluster centroid locations. Then, the query cells are modeled using the Symphony 759 mixture model and corrected to their approximate locations in the integrated embedding by regressing 760 out the query batch-dependent effects (Algorithm 2).

\section{Projection of query cells into pre-harmonized PC Space}

762 Symphony projects the query cells into the same original PCs $\left(Z_{r}\right)$ as the reference. Symphony 763 assumes that, given a much smaller query compared to the reference $(m<<n)$, the PCs will remain 
764 approximately stable with the addition of query cells. To project the query cells, we first subset the

765 query expression data by the same variable genes used in reference building and scale the normalized 766 expression of each gene by the same mean and standard deviations used to scale the reference cells

$767(\mu, \sigma)$. Let $G_{q s}$ denote the query gene expression matrix scaled by the reference gene means and

768 standard deviations. We can then use the reference gene loadings $(U)$ to project $G_{q s}$ into reference PC

769 space. In (4), $Z_{q} \in \mathbb{R}^{d \times m}$ denotes the PC embedding for the query cells. Note that if an alternate

770 starting embedding (e.g. CCA) is used instead of PCA, the gene loadings must be saved to enable this

771 query projection step.

$$
Z_{q}=U^{T} G_{q s}=\Sigma_{q} V_{q}^{T}
$$

\section{Soft assignment across reference clusters}

773 Once the query cells are projected into PC space, we soft assign the cells to the reference clusters

774 using the saved reference centroid locations $\left(Y_{\cos }\right)$. Symphony assumes that the reference cluster

775 centroid locations remain approximately stable with the addition of a much smaller query dataset since

776 the query contains no novel cell types. Under these conditions, we use a previously published objective

777 function for soft $k$-means clustering (5), which includes a distance term and an entropy regularization

778 term over $R$ weighted by hyperparameter $\sigma$. This is the same objective function as the clustering step of

779 Harmony, except it does not include the diversity penalty term. In Harmony, the purpose of the diversity

780 term is to penalize clusters that are only represented by one or a few datasets (suggesting they do not

781 represent true cell types). In contrast, Symphony does not require the use of a diversity penalty

782 because the reference centroids have already been established. Furthermore, the query cell types can

783 comprise a subset of a larger set of reference cell types, and therefore not all clusters are necessarily

784 expected to be represented in the query. We can solve for $R_{q}$, the optimal probabilistic assignment for

785 query cells across each of the $k$ reference clusters (Implementation Details).

$$
\min _{R, Y} \sum_{i, k} R_{k i}\left\|Z_{i}-Y_{k}\right\|^{2}+\sigma R_{k i} \log R_{k i}
$$




$$
\text { s.t. } \forall_{i} \forall_{k} R_{k i}>0, \forall_{i} \sum_{k=1}^{K} R_{k i}=1
$$

\section{Mixture of experts correction}

787 The final step in Symphony mapping is to model then remove the query batch effects to obtain $\hat{Z}_{q}$, the

788 approximate location of query cells in the harmonized reference embedding. In equation (2), we

789 modeled the reference and query cells together and wish to solve for the query parameters $B_{q k} \in$

$790 \mathbb{R}^{(1+c) \times d}$ for each cluster $k$. The reference-dependent terms in (2) were previously computed and

791 saved in compressed form $\left(N_{r}\right.$ and $\left.C\right)$. With $R_{q}$ and $Z_{q}$ calculated from query cell projection and

792 clustering, we can finally solve for $B_{q k}$. Similar to the correction step of Harmony, we obtain cell-specific

793 correction values for the query cells by removing the batch-dependent terms captured in $B_{q k[1: c, j]}$. Note

794 that the reference batch terms are neither modeled nor corrected during reference mapping, so the

795 harmonized reference cells do not move.

796 The final locations of the query cells in the harmonized embedding are estimated by iterating over all $k$ 797 clusters and subtracting out the non-intercept batch terms for each cell weighted by cluster membership

798 (6). Intuitively, the query centroids are moved so that they overlap perfectly with the reference centroids

799 in the harmonized embedding. $\hat{Z}_{q[i]}$ denotes the approximate location in harmonized PC space for

800 query cell $i$.

$$
\begin{gathered}
Z_{q[i]}=\sum_{k} R_{q[k, i]}\left[B_{q k[0, \cdot]}^{T}+B_{q k[1: c, i]}^{T} X_{q}\right]+\varepsilon \\
\hat{Z}_{q[i]}=Z_{q[i]}-\sum_{k} R_{q[k, i]} B_{q k[1: c, j]}^{T} X_{q} \\
\hat{Z}_{q[i]}=\sum_{k} R_{q[k, i]} B_{q k[0, \cdot]}^{T}+\varepsilon
\end{gathered}
$$

801 Algorithm 2 Map query cells onto reference

802 function QUERYMAPPING $\left(G_{q}, X_{q}, \operatorname{Ref}\right)$

803

$$
G_{q s} \leftarrow \operatorname{SCALE}\left(G_{q}, \operatorname{Ref} \$ \mu, \operatorname{Ref} \$ \sigma\right) \quad \triangleright \$ \text { denotes accessing a component of Ref }
$$




$$
Z_{q} \leftarrow \text { PCAPROJECTION }\left(G_{q S}, \operatorname{Ref} \$ U\right)
$$

$$
R_{q} \leftarrow \operatorname{ClUSteR}\left(Z_{q}, \operatorname{Ref} \$ Y_{\text {cos }}\right)
$$

$$
\hat{Z}_{q} \leftarrow Z_{q}
$$

\section{6}

\section{$817 \quad 1.5$ Implementation details}

\section{Reference building and compression}

\section{Variable gene selection and scaling}

820 Starting with the gene expression matrix for reference cells, we perform log(CP10K) library size

821 normalization of the cells (if not already done), subset by the top $g$ variable genes by the vst method 822 (as provided in Seurat ${ }^{18}$ ), which fits a line to the log(variance) and log(mean) relationship using local 823 polynomial regression, then standardizes the features by observed mean and expected variance, 824 calculating gene variance on the standardized values, which is re-implemented as a standalone 825 function at https://github.com/immunogenomics/singlecellmethods. The data is scaled such that the expression of each gene has a mean expression of 0 and variance of 1 across all cells.

\section{PCA}

828 We perform dimensionality reduction on the scaled gene expression $G_{r s}$ using principal component 829 analysis (PCA). PCA projects the data a low-dimensional, orthonormal embedding that retains most of 
830 the variation of gene expression in the dataset. Singular value decomposition (SVD) is a matrix

831 factorization method that can calculate the PCs for a dataset. Here, we use SVD (irlba package in $\mathrm{R}^{53}$ )

832 to perform PCA. SVD states that matrix $G_{r s}$ with dimensions $g \times n$ can be factorized as:

$$
G_{r s}=U \Sigma V^{T}
$$

$833 \operatorname{In}(7), \Sigma V^{T}=Z_{r}$ (dimensions $d \times n$ ) represents the embedding of reference cells in PC space, after

834 truncating the matrix on the first $d$ (by default, $d=20)$ PCs. The gene loadings $\left(U \in \mathbb{R}^{g \times d}\right)$ are saved.

835 Note that an alternative embedding, such as canonical correlation analysis (CCA) may be used in place 836 of PCA, as long as the gene loadings are saved.

\section{Harmony integration}

838 The PCA embedding $\left(Z_{r}\right)$ is then input to Harmony for dataset integration. By default, Symphony uses 839 the default parameters for the cluster diversity enforcement $(\theta=2)$, the entropy regularization

840 hyperparameter for soft $k$-means $(\sigma=0.1)$, and the number of clusters $k=\min \left(100, \frac{n}{30}\right)$. We save the 841 L2-normalized cluster centroid locations $Y_{c o s}$ to the reference object since query mapping employs a 842 cosine distance metric. If the reference has a single-level batch structure, no integration is performed, 843 and the clusters are defined using soft k-means.

\section{Query mapping}

\section{$845 \quad$ Normalization and scaling}

846 The gene expression for query cells are assumed to be library size normalized in the same manner that 847 was used to normalize the reference cells (e.g. $\log (\mathrm{CP} 10 \mathrm{~K}))$. During scaling, the query data is subset 848 by the same variable genes from the reference datasets, and query gene expression is scaled by the 849 reference gene means and standard deviations. Any genes present in the query but not the reference 850 are ignored, and any genes present in the reference but not the query have scaled expression set to 0.

\section{Clustering step uses cosine distance}


852 As in Harmony, in practice we use cosine distance rather than Euclidean distance in the clustering step.

853 For the computation of the distance term, we L2-normalize the columns (cells) of $Z$ and columns

854 (centroids) of $Y_{k}$ such that the squared values sum to 1 across each column. Let the terms $Z_{q_{-} \cos [\cdot, i]}$ and

$855 Y_{\cos [;, \mathrm{k}]}$ represent the L2-normalized locations of query cell $i$ and the reference centroid for cluster $k$ in

856 PC space, respectively. We compute the cosine distance between the cells and centroids. Since all

$857 Z_{q_{-} \cos [;, i]}$ and $Y_{\cos [;, \mathrm{k}]}$ each have unity norm, the squared Euclidean distance $\left\|Z_{q_{-} \cos [;, i]}-Y_{\cos [;, \mathrm{k}]}\right\|^{2}$ is

858 equivalent to the cosine distance $2\left(1-\cos \left(Y_{\cos [;, \mathrm{k}]}, Z_{q_{-} \cos [;, i]}\right)\right)=2\left(1-Y_{\cos \left[k,{ }^{\prime}\right]}^{T} Z_{q_{-} \cos [;, i]}\right)$. Therefore, the

859 objective function for query assignment to centroids becomes:

$$
\begin{gathered}
\min _{R, Y} \sum_{i, k} 2 R_{q[k, i]}\left(1-Y_{\cos [k, \cdot]}^{T} Z_{q_{-} \cos [\cdot, i]}\right)+\sigma R_{q[k, i]} \log R_{q[k, i]} \\
\text { s.t. } \forall_{i} \forall_{k} R_{q[k, i]}>0, \forall_{i} \sum_{k=1}^{K} R_{q[k, i]}=1
\end{gathered}
$$

860 We can solve the optimization problem using an expectation-maximization framework. Following the 861 same strategy as Korsunsky et al. (2019), we calculate $R_{i}$, the optimal probabilistic assignment for each 862 query cell $i$ across each of the $k$ reference clusters. In (9), we can interpret $R_{q[k, i]}$ as the probability that 863 query cell $i$ belongs to cluster $k$. The denominator term simply ensures that for any given cell $i$, the 864 probabilities across all $k$ clusters sum to one. By default, sigma $=0.1$

$$
R_{q(k, i)}=\frac{\exp \left(-\frac{2}{\sigma}\left(1-Y_{\cos [k, \cdot]}^{T} Z_{q_{-} \cos [;, i]}\right)\right)}{\sum_{k=1}^{K} \exp \left(-\frac{2}{\sigma}\left(1-Y_{\cos [k, \cdot]}^{T} Z_{q_{-} \cos [;, i]}\right)\right)}
$$

\section{2. Analysis details}

866

$$
2.1 \text { 10x PBMCs analysis }
$$

\section{Preprocessing scRNA-seq data}


868 The three 10x PBMCs datasets were previously preprocessed by our group as part of the Harmony 869 publication. We used the same $\log (1+\mathrm{CP} 10 \mathrm{~K})$ normalized expression data, filtered as described in 870 Korsunsky et al. $(2019)^{17}$. The PBMCs consist of cells from three technologies: 3'v1 ( $n=4,808$ cells),

871 3'v2 (8,372 cells), and 5' (7,612 cells).

\section{Symphony mapping experiments}

873 To construct each of three references for subsequent mapping, we aggregated two reference datasets

874 into a single normalized expression matrix and identified the top 2,000 variable genes across all cells 875 using the variance stabilizing transformation (VST) procedure ${ }^{18}$. We ran Harmony on the top 20 PCs 876 and default 100 clusters, harmonizing over 'technology' with default parameters. For Symphony 877 mapping, we specified query ‘technology’ covariate.

\section{Constructing gold standard embedding}

879 To construct the gold standard de novo Harmony embedding, we concatenated all three datasets 880 together into a single expression matrix, subsetted by the top 2,000 variable genes over all cells, and 881 ran Harmony integration on the top 20 PCs, harmonizing over 'technology' with default parameters.

\section{Assigning ground truth cell types}

883 We clustered the cells in the gold standard embedding using the Louvain algorithm as implemented in 884 the Seurat functions BuildSNN and RunModularityClustering ${ }^{18}$. For PBMCs, we used $\mathrm{nn} \_\mathrm{k}=5$ (to 885 capture rare HSCs), nn_eps $=0.5$, and resolution $=0.8$. We labeled clusters with ground truth cell types 886 according to expression of canonical lineage marker genes (Table S2). PBMCs were assigned across 8877 types: T (CD3D), NK (GNLY), B (MS4A1), Monocytes (CD14, FCGR3A), DCs (FCER1A), 888 Megakaryocytes (PPBP), and HSCs (CD34). Clusters were labeled if the AUC (calculated using 889 presto $^{54}$ ) for the corresponding lineage marker was $>0.62$. For clusters that did not express a specific 890 lineage marker, we manually assigned a cell type based on the top differentially expressed genes 891 (Table S2). PBMCs cluster 20 was identified as low-quality cells (high in mitochondrial genes; Table 
892 S2). We removed all cells in this cluster $(n=94)$ from further analyses. The final ground truth labels were 893 used in downstream analyses and cell type classification accuracy evaluation.

\section{Evaluation of mixing and cell type classification accuracy}

895 To compare dataset mixing between de novo integration and mapping, we calculated Local Inverse 896 Simpson Index (LISI) using the compute_lisi function from https://github.com/immunogenomics/LISI. 897 For each mapping experiment, we calculated dataset LISI on all cells, then subsetted the results for 898 query cell neighborhoods only to measure the effective number of datasets in the local neighborhood of 899 each query cell.

900 We predicted query cell types by transferring reference cell type annotations using the knn function in 901 the 'class' $\mathrm{R}$ package $(\mathrm{k}=5)$. We calculated overall accuracy across all query cells and cell type $\mathrm{F} 1$ 902 scores (the harmonic mean of precision and recall, ranging from 0 to 1 ). Precision $=T P /(T P+F P)$, recall $903=\mathrm{TP} /(\mathrm{TP}+\mathrm{FN}), \mathrm{F} 1=(2$ * precision * recall $) /($ precision + recall $)$. Cell type $\mathrm{F} 1$ was the metric Abdelaal et 904 al. used to benchmark automated cell type classifiers ${ }^{35}$. We used their evaluate. $R$ script to calculate 905 confusion matrices and F1 scores by cell type.

\section{Quantifying local similarity between two embeddings}

$907 \mathrm{k}-\mathrm{NN}$-correlation ( $\mathrm{k}-\mathrm{NN}$-corr) is a new metric that quantifies how well a given alternative embedding 908 preserves the local neighborhood structure with respect to a gold standard embedding. Anchoring on 909 each query cell, we calculate (1) the pairwise similarities to its $k$ nearest reference neighbors in the gold 910 standard embedding and (2) the similarities between the same query-reference neighbor pairs in an 911 alternate embedding (Methods), then calculate the Spearman (rank-based) correlation between (1) 912 and (2). For similarity, we use the radial basis function kernel: $\operatorname{similarity}(\mathrm{x}, \mathrm{y})=\exp \left(-\|\mathrm{x}-\mathrm{y}\|^{2} /\left(2 \sigma^{2}\right)\right)$. For 913 each query cell, we obtain a single k-NN-corr value capturing how well the relative similarities to its $k$ 914 nearest reference neighbors are preserved. Note that k-NN-corr is asymmetric with respect to which 915 embedding is selected as the gold standard and which is selected as the alternative because the 916 nearest neighbor pairs are fixed based on how they were defined in the gold standard. The distribution 
917 of k-NN-corr scores for all query cells can measure the embedding quality, where higher k-NN-corr

918 indicates greater recapitulation of the gold standard. Lower values for $k$ assess more local

919 neighborhoods, whereas higher $k$ assesses more global structure.

920 We calculated k-NN-corr between the gold standard Harmony embedding and two alternative

921 embeddings: (1) the full Symphony mapping algorithm (projection, clustering, and correction) and (2)

922 PCA-projection only as a comparison to a batch-naïve mapping. PCA-projection refers to the first step

923 of Symphony mapping, where query cells are projected from gene expression to pre-harmonized PC

924 space: $Z_{q}=U^{\top} G_{q}$.

9252.2 Benchmarking against automatic cell type classifiers

926 We downloaded the PbmcBench benchmarking dataset used by a recent comparison of automatic cell 927 type identification methods ${ }^{35,39}$. For each of 48 train-test experiments previously described ${ }^{35}$, we used 928 the same evaluation metrics (median cell type F1 score) to evaluate Symphony in comparison to the 22 929 other classifiers. We obtained the numerical F1-score results for the other classifiers for all 48

930 experiments directly from the authors in order to determine Symphony's place within the rank ordering 931 of classifier performance.

932 During reference building, we explored two different gene selection methods: (1) unsupervised (top 9332000 variable genes) and (2) supervised based on identifying the top 20 differentially expressed (DE) 934 genes per cell type. Option (2) was included to give Symphony the same information as prior-

935 knowledge classifiers (e.g. SCINA with 20 marker genes per cell type). We used the 'presto' package ${ }^{54}$ 936 for DE analysis. No integration was performed because the reference had a single-level batch structure 937 (clusters were simply assigned using soft k-means). Onto each of 7 references (each representing 1 938 protocol for donor pbmc1), we mapped either a second protocol for donor pbmc1 (6 experiments) or the 939 same protocol for donor pbmc2 (1 experiment). Given the resulting Symphony joint feature 940 embeddings, we used three downstream classifiers to predict query cell types: 5-NN, SVM with a radial 941 kernel, and glm_net with ridge ${ }^{55}$. A total of 6 Symphony-based classifiers were tested (2 gene selection 942 methods * 3 downstream classifiers). 


\subsection{Pancreas benchmark}

\section{Constructing the pancreas query with mouse and human}

945 The pancreas query dataset (Baron et al., 2016; inDrop, $n=8,569$ human and 1,886 mouse cells) along

946 with author-defined cell type labels were downloaded from https://hemberg-

947 lab.github.io/scRNA.seq.datasets/human/pancreas/. In order to combine the human and mouse

948 matrices into a single aggregated query, we "humanized" the mouse expression matrix by mapping 949 mouse genes to their orthologous human genes. This mapping was computed using the biomaRt $\mathrm{R}$ 950 package ${ }^{56}$, mapping mgi_symbol from the mmusculus_gene_ensembl database to hgnc_symbol 951 from the hsapien_gene_ensembl database. We added additional ortholog pairs from HomoloGene

952 (https://ftp.ncbi.nih.gov/pub/HomoloGene/build37.2/homologene.data) to obtain a total of 22,578 human 953 to mouse gene ortholog pairs. We represented this map as a matrix, with mouse genes as rows, human 954 genes as columns, and values in $\{0,1\}$ assigned to denote whether a mouse gene maps to a human 955 gene. We then normalized the matrix to have each column sum to one, effectively creating a count956 preserving probabilistic map from d mouse to $D$ human genes $M \in R^{D \times d}$. Mapping from mouse to 957 human genes is then performed with matrix multiplication: $U_{\text {human }}=M_{\text {mouse. }}$ Note that while the mouse 958 gene expression matrix $U_{\text {mouse }}$ contains only integers $\left(\cup_{\text {mouse }} \in Z^{\mathrm{d} \times N}\right)$, the many-to-many mapping means 959 that the mapped human gene expression matrix $U_{\text {human }}$ may contain non-integers $\left(U_{\text {human }} \in \mathrm{R}^{\mathrm{D} \times \mathrm{N}}\right)$. For 960 any human orthologs that were missing in the mouse expression data, we filled in the expression with 961 zeroes. We then $\log (\mathrm{CP} 10 \mathrm{~K}+1)$ normalized the query cells.

\section{Preprocessing reference scRNA-seq data}

963 The pancreas reference datasets were each sequenced with a different technology: Fluidigm C1 964 ( $n=638$ cells), CEL-seq (946 cells), CEL-seq2 (2,238 cells), Smart-seq2 (2,355 cells). We obtained the $965 \log (1+\mathrm{CP} 10 \mathrm{~K})$ normalized data from the Harmony publication ${ }^{17}$. The pancreas cells were previously 966 assigned across 9 types within each dataset individually according to cluster-specific expression of 967 marker genes: alpha (GCG), beta (MAFA), gamma (PPY), delta (SST), acinar (PRSS1), ductal 
968 (KRT19), endothelial (CDH5), stellate (COL1A2), and immune (PTPRC). We removed 290 cells that

969 were left unassigned as part of ambiguous or outlier clusters during within-dataset annotation, leaving $970 \quad 5,887$ reference cells.

971 We benchmarked three reference mapping methods as follows:

\section{Symphony mapping onto a Harmony reference}

973 We calculated the top 1,000 variable genes within each of the four reference dataset separately using

974 VST then pooled them (total 2,236 variable genes) for PCA. For reference integration, we ran Harmony 975 on the top 20 PCs, harmonizing over 'donor' $(\theta=2)$ and 'technology' $(\theta=4)$, with $\tau=5$. For Symphony 976 mapping, we specified query 'donor', 'species', and 'technology' covariates.

977 As a comparison with de novo integration, we ran Harmony integration on all 5 datasets together. We 978 pooled the top 1,000 variable genes within each dataset (total 2,650 genes), calculated the top 20 PCs, 979 and harmonized over 'species' $(\theta=2)$, 'donor' $(\theta=2)$, and 'technology' $(\theta=2)$.

$980 \quad$ Seurat $\mathbf{4}$ mapping onto a Seurat reference

981 We ran Seurat version 4 (beta) $)^{30}$ (Seurat_3.9.9.9024) and followed the steps from the author's tutorial 982 (https://satijalab.org/seurat/v3.2/integration.html) to integrate the reference datasets given that the 983 FindIntegrationAnchors and IntegrateData functions for de novo integration are equivalent between 984 Seurat v3 and v4 to our understanding. We used the same 2,236 variable genes as above and 20 PCs. 985 We followed the tutorial (https://satijalab.org/seurat/v4.0/reference mapping.html) to map each donor 986 dataset from the query individually. We used the FindTransferAnchors function with reduction = 987 'pcaproject' and MapQuery function with reference.reduction = 'pca' (as the documentation 988 recommends for unimodal analysis).

989 As a comparison with de novo integration, we ran Seurat v3/4 integration (FindlntegrationAnchors and 990 IntegrateData) on all 5 datasets (integrating over plate-based technologies and Baron donors as 991 batches) with the same 2,650 variable genes as above. 
scArches mapping onto a trVAE reference

993 We ran scArches ${ }^{28}$ version 0.3 with $\operatorname{trVAE}^{33}$ using default parameters provided in the authors'

994 notebooks (https://github.com/theislab/scarches/tree/master/notebooks). For the pancreas analysis, we

995 only had access to normalized expression data and therefore ran scArches with trVAE using the mse

996 reconstruction loss function. We included query batch information in the condition_key parameter.

997 As a comparison with de novo integration, we ran trVAE on all 5 datasets with default parameters,

998 specifying batch as 'dataset' for the 4 plate-based datasets and 'donor' for the Baron et al. dataset.

\section{Evaluation metrics}

000 We used the resulting joint (reference and query) cell embedding to predict query cell types from 001 reference cells using a 5-NN classifier and calculated cell type prediction F1 scores, as described 002 above. Note that for the cell type prediction and cell type F1 score calculation, we excluded query 003 Schwann cells from the accuracy metrics because that cell type is not present in the reference.

004 To assess degree of mixing, we calculated ref_query LISI and query donor LISI on query cell 005 neighborhoods using the compute_lisi function as above. ref_query LISI measures how well the 006 reference and query datasets are mixed (max ref_query LISI = 2), whereas query donor LISI measures 007 how well the individual donors within the query dataset are mixed $(\max =6)$.

008 We measured mapping runtime and corresponding de novo integration runtime for each method as 009 elapsed time starting from gene expression. Symphony and Seurat were run in interactive Jupyter 010 notebooks on a Linux server (Intel Xeon E5-2690 v.3 processors), whereas scArches/trVAE was run on 011 GPUs (graphics card GP100GL [Tesla P100 PCle 16GB]) to speed up runtime.

\section{$012 \quad$ 2.3 Fetal liver hematopoiesis trajectory inference example}

013 We obtained post-filtered, post-doublet removal data directly from the authors ${ }^{46}$ along with author014 defined cell type annotations for 113,063 cells sequenced with $10 \times 3$ ' end bias and a separate 25,367 015 cells sequenced with 10x 5' end bias. For building the harmonized reference from all 3' cells, we 
016 followed the same variable gene selection procedures as the original authors, using the Seurat

017 variance/mean ratio (VMR) method with parameters min_expr $=.0125$, max_expr $=3$, and

018 min_dispersion $=0.625$ (resulting in 1,917 variable genes). For each of 14 held-out donor experiments

019 within the 3' dataset, we integrated the reference with Harmony on 13 donors $(\theta=3)$. During Symphony

020 mapping, we specified query 'donor' covariate. For mapping 5' cells against a 3' reference, we removed

021 two donors (F2 and F5, n=3,953) from the 5' query based on low library complexity (Fig. S5b), leaving

$022 \mathrm{n}=21,414$ cells from 5 donors. We integrated the reference (all 14 donors sequenced with 3' end bias)

023 with Harmony over 'donor' $(\theta=3)$. During Symphony mapping, we specified both 'donor' and

024 'technology' as covariates. We predicted query cell types by transferring reference cell type annotations

025 using the knn function in the 'class' R package $(k=30)$. We visualized the aggregated confusion matrix

026 across all 14 held-out donor experiments as well as the confusion matrix for the single 5'-to-3'

027 experiment using ComplexHeatmap R package ${ }^{57}$.

028 For the trajectory inference analysis, we obtained trajectory coordinates from the force directed graph

029 (FDG) embedding of all 3'-sequenced cells from the original authors ${ }^{46}$, forming a reference trajectory.

030 We restricted the trajectory to immune cell types only (excluding hepatocytes, fibroblasts, and

031 endothelial). We then mapped a subset of the query cells belonging to the MEM lineage (MEMPs,

032 megakaryocytes, mast cells, early-late erythroid; $n=5,141$ ) to the reference-defined trajectory by

033 averaging the FDG coordinates of the 10 reference immune cell neighbors in the Symphony

034 embedding.

0352.4 Memory T cell surface protein inference example

036 We used a memory T cell CITE-seq dataset collected from a tuberculosis disease progression cohort of

037259 individuals of admixed Peruvian ancestry ${ }^{40}$. The dataset includes expression of the whole

038 transcriptome (33,538 genes) and 30 surface protein markers from 500,089 memory T cells isolated

039 from PBMCs. Including technical replicates, 271 samples were processed across 46 batches.

040 To assess protein prediction accuracy using Symphony embeddings, we randomly selected 217

041 samples (411,004 cells), normalized the expression of each gene (log2(CP10K)) and built a Symphony 
042 reference based on mRNA expression, correcting for donor and batch. The held-out 54 samples

043 comprised the query that we mapped onto the reference. We predicted the expression of each of the 30

044 surface proteins in each of the query cells by averaging the protein's expression across the cell's 50

045 nearest reference neighbors. Nearest neighbors were defined based on Euclidean distance in the

046 batch-corrected low-dimensional embedding. As a ground truth for each protein in each query cell, we

047 computed a smoothed estimate of the cells' measured protein expression by averaging the protein's

048 expression across the cell's 50 nearest neighbors in the batch-corrected complete PCA embedding of

049 all 259 donors. We did not use the cells' raw measured protein expression due to dropout. We

050 computed the Pearson correlation coefficient between our predicted expression and the ground truth

051 expression across all cells per donor for each marker.

052 To assess protein prediction accuracy based on mapping to a joint mRNA and protein-based

053 Symphony reference, we first built an integrated reference by using canonical correlation analysis

054 (CCA) to project cells into a low-dimensional embedding maximizing correlation between mRNA and 055 protein features. We randomly selected 217 samples (395,373 cells) to comprise this reference, and 056 normalized the expression of each gene $(\log 2(\mathrm{CP} 10 \mathrm{~K}))$, selected the top 2,865 most variable genes, 057 and scaled (mean $=0$, variance $=1$ ) all mRNA and protein features. We computed 20 canonical 058 variates (CVs) with the cc function in the CCA R package ${ }^{58}$ and corrected the mRNA CVs for donor and 059 batch effects with Harmony. Then, we used Symphony to construct a reference based on the batch-

060 corrected CVs, gene loadings on each CV, and mean and standard deviation used to scale each gene 061 prior to CCA. The held-out 54 samples comprised the query that we mapped onto the reference. As 062 described above, we predicted the expression of each of the 30 surface proteins in each of the query 063 cells based on the cell's 5, 10, or 50 nearest neighbors in the reference, estimated the smoothed 064 ground truth expression of each protein in each query cell (now based on the batch-corrected CCA 065 embedding of all 259 donors) and computed the Pearson correlation coefficient for each marker. 
067 For visualizing the embeddings using UMAP $^{59}$ (and included as the default in Symphony), we used the

068 'uwot' R package with the following parameters: $n$ _neighbors=30, learning_rate=0.5, init = 'laplacian', 069 metric $=$ 'cosine', min_dist=0.1 (except min_dist=0.3 for pancreas and fetal liver examples). For each

070 Symphony reference, we saved the uwot model at the time of UMAP using the uwot::save_uwot

071 function and saved the path to the model file as part of the Symphony reference object. Saving the

072 reference UMAP model allows for the fast projection of new query cells into reference UMAP space

073 from the query embedding from Symphony mapping using the function uwot::transform.

074 For the pancreas benchmarking, we computed a de novo UMAP embedding on the joint reference and 075 query embedding because a UMAP projection can potentially obscure differences between the 076 projected data and dataset used to construct the UMAP model. For general purposes, we recommend 077 UMAP projection when the reference cell UMAP coordinates are desired to remain stable.

078 To distinguish the reference plots from query plots, we visually present the reference embedding as a 079 contour density instead of individual cells. The density plots were generated using ggplot2 function 080 stat_density_2d with geom = 'polygon' and contour_var = 'ndensity'. We provide custom functions to 081 generate these plots as part of the Symphony package.

\section{$082 \quad 2.6$ Runtime scalability analysis}

083 We downsampled a large memory T cell dataset ${ }^{40}$ to create benchmark reference datasets with 20,000, $08450,000,100,000,250,000$, and 500,000 cells. For each, we built a reference (20 PCs, 100 centroids) 085 integrating over 'donor' and mapped three different-sized queries: 1,000, 10,000, and 100,000 cells. To 086 isolate the separate effects of number of query cells and number of query batches on mapping time, we 087 mapped against the 50,000-cell reference: (1) varying the number of query cells (from 1,000 to 10,000 088 cells) while keeping the number of donors constant and (2) varying the number of query donors (6 to 089120 donors) while keeping the number of cells constant (randomly sampling 10,000 cells). We also 090 performed separate experiments varying the number of reference centroids (25 to 400 ) and number of 091 dimensions (10 to 320 PCs) while keeping all other parameters constant. We ran all jobs on Linux 
092 servers allotted 4 cores and 64 GB of memory (Intel Xeon E5-2690 v.3 processors) and used the 093 system.time R function to measure elapsed time.

\section{Data availability}

095 Datasets for all analyses were obtained from the links in Table S1. All datasets are publicly available 096 except the memory T cell CITE-seq data, which will be available at GEO accession GSE158769.

\section{Code availability}

098 We provide an efficient implementation of Symphony at https://github.com/immunogenomics/symphony

099 along with documentation, tutorials, and pre-built references. Scripts reproducing figures for all

100 examples will be made available at https://github.com/immunogenomics/symphony reproducibility.

\section{References}

102 1. Klein, A. M. \& Treutlein, B. Single cell analyses of development in the modern era. Development 103 146, (2019).

104 2. Han, X. et al. Construction of a human cell landscape at single-cell level. Nature (2020)

105 doi:10.1038/s41586-020-2157-4.

106 3. Svensson, V., da Veiga Beltrame, E. \& Pachter, L. A curated database reveals trends in single-cell 107 transcriptomics. Database 2020, (2020).

108 4. Cao, J. et al. The single-cell transcriptional landscape of mammalian organogenesis. Nature 566, 109 496-502 (2019).

110 5. Jerber, J. et al. Population-scale single-cell RNA-seq profiling across dopaminergic neuron

111 differentiation. bioRxiv 2020.05.21.103820 (2020) doi:10.1101/2020.05.21.103820.

112 6. Zhang, F. et al. Defining inflammatory cell states in rheumatoid arthritis joint synovial tissues by 113 integrating single-cell transcriptomics and mass cytometry. Nat. Immunol. 20, 928-942 (2019).

114 7. Reyes, M. et al. An immune-cell signature of bacterial sepsis. Nat. Med. 26, 333-340 (2020). 
115 8. Kotliarov, Y. et al. Broad immune activation underlies shared set point signatures for vaccine responsiveness in healthy individuals and disease activity in patients with lupus. Nat. Med. 26,

9. Schafflick, D. et al. Integrated single cell analysis of blood and cerebrospinal fluid leukocytes in multiple sclerosis. Nat. Commun. 11, 247 (2020).

10. Smillie, C. S. et al. Intra- and Inter-cellular Rewiring of the Human Colon during Ulcerative Colitis. Cell 178, 714-730.e22 (2019).

11. Litviňuková, M. et al. Cells of the adult human heart. Nature (2020) doi:10.1038/s41586-020-27974.

12. Rozenblatt-Rosen, O., Stubbington, M. J. T., Regev, A. \& Teichmann, S. A. The Human Cell Atlas: from vision to reality. Nature 550, 451-453 (2017).

13. Haghverdi, L., Lun, A. T. L., Morgan, M. D. \& Marioni, J. C. Batch effects in single-cell RNAsequencing data are corrected by matching mutual nearest neighbors. Nat. Biotechnol. 36, 421-

14. Hie, B., Bryson, B. \& Berger, B. Efficient integration of heterogeneous single-cell transcriptomes using Scanorama. Nat. Biotechnol. 37, 685-691 (2019).

15. Welch, J. D. et al. Single-Cell Multi-omic Integration Compares and Contrasts Features of Brain Cell Identity. Cell 177, 1873-1887.e17 (2019).

16. Lopez, R., Regier, J., Cole, M. B., Jordan, M. I. \& Yosef, N. Deep generative modeling for singlecell transcriptomics. Nat. Methods 15, 1053-1058 (2018).

17. Korsunsky, I. et al. Fast, sensitive and accurate integration of single-cell data with Harmony. Nat. Methods 16, 1289-1296 (2019).

18. Stuart, T. et al. Comprehensive Integration of Single-Cell Data. Cell 177, 1888-1902.e21 (2019).

138 19. He, Z., Brazovskaja, A., Ebert, S., Camp, J. G. \& Treutlein, B. CSS: cluster similarity spectrum 139 integration of single-cell genomics data. Genome Biol. 21, 224 (2020).

140 20. Tran, H. T. N. et al. A benchmark of batch-effect correction methods for single-cell RNA sequencing data. Genome Biol. 21, 12 (2020). 
142 21. Zhang, Q. et al. Landscape and Dynamics of Single Immune Cells in Hepatocellular Carcinoma. Cell 179, 829-845.e20 (2019).

22. Wei, K. et al. Notch signalling drives synovial fibroblast identity and arthritis pathology. Nature $\mathbf{5 8 2}$, 259-264 (2020).

23. Kirita, Y., Wu, H., Uchimura, K., Wilson, P. C. \& Humphreys, B. D. Cell profiling of mouse acute kidney injury reveals conserved cellular responses to injury. Proc. Natl. Acad. Sci. U. S. A. 117,

27. Lähnemann, D. et al. Eleven grand challenges in single-cell data science. Genome Biol. 21, 31 15874-15883 (2020).

24. Sandu, I. et al. Landscape of Exhausted Virus-Specific CD8 T Cells in Chronic LCMV Infection. Cell Rep. 32, 108078 (2020).

25. Korsunsky, I. et al. Cross-tissue, single-cell stromal atlas identifies shared pathological fibroblast phenotypes in four chronic inflammatory diseases. bioRxiv 2021.01.11.426253 (2021).

26. Zhang, F. et al. IFN- $y$ and TNF- $\alpha$ drive a CXCL10 + CCL2 + macrophage phenotype expanded in severe COVID-19 and other diseases with tissue inflammation. bioRxiv (2020) doi:10.1101/2020.08.05.238360. (2020).

28. Lotfollahi, M. et al. Query to reference single-cell integration with transfer learning. bioRxiv (2020).

29. Cao, Z.-J., Wei, L., Lu, S., Yang, D.-C. \& Gao, G. Searching large-scale scRNA-seq databases via unbiased cell embedding with Cell BLAST. Nat. Commun. 11, 3458 (2020).

30. Hao, Y. et al. Integrated analysis of multimodal single-cell data. bioRxiv 2020.10.12.335331 (2020).

31. Kiselev, V. Y., Yiu, A. \& Hemberg, M. scmap: projection of single-cell RNA-seq data across data sets. Nat. Methods 15, 359-362 (2018).

32. Xu, C. et al. Probabilistic harmonization and annotation of single-cell transcriptomics data with deep generative models. Mol. Syst. Biol. 17, e9620 (2021).

33. Lotfollahi, M., Naghipourfar, M., Theis, F. J. \& Wolf, F. A. Conditional out-of-distribution generation for unpaired data using transfer VAE. Bioinformatics 36, i610-i617 (2020). 
168 34. Polański, K. et al. BBKNN: fast batch alignment of single cell transcriptomes. Bioinformatics 36, 964-965 (2020).

35. Abdelaal, T. et al. A comparison of automatic cell identification methods for single-cell RNA sequencing data. Genome Biol. 20, 194 (2019).

36. Zhang, Z. et al. SCINA: A Semi-Supervised Subtyping Algorithm of Single Cells and Bulk Samples. Genes 10, (2019).

37. Alquicira-Hernandez, J., Sathe, A., Ji, H. P., Nguyen, Q. \& Powell, J. E. scPred: accurate supervised method for cell-type classification from single-cell RNA-seq data. Genome Biol. 20, 264

39. Ding, J. et al. Systematic comparative analysis of single cell RNA-sequencing methods. bioRxiv 632216 (2019) doi:10.1101/632216.

40. Nathan, A. et al. Multimodal memory T cell profiling identifies a reduction in a polyfunctional Th17 state associated with tuberculosis progression. bioRxiv 2020.04.23.057828 (2020) doi:10.1101/2020.04.23.057828.

41. Segerstolpe, Å. et al. Single-Cell Transcriptome Profiling of Human Pancreatic Islets in Health and Type 2 Diabetes. Cell Metab. 24, 593-607 (2016).

42. Lawlor, N. et al. Single-cell transcriptomes identify human islet cell signatures and reveal cell-typespecific expression changes in type 2 diabetes. Genome Res. 27, 208-222 (2017).

43. Grün, D. et al. De Novo Prediction of Stem Cell Identity using Single-Cell Transcriptome Data. Cell Stem Cell 19, 266-277 (2016).

44. Muraro, M. J. et al. A Single-Cell Transcriptome Atlas of the Human Pancreas. Cell Syst 3, 385394.e3 (2016).

45. Baron, M. et al. A Single-Cell Transcriptomic Map of the Human and Mouse Pancreas Reveals Inter- and Intra-cell Population Structure. Cell Syst 3, 346-360.e4 (2016).

194 46. Popescu, D.-M. et al. Decoding human fetal liver haematopoiesis. Nature 574, 365-371 (2019). 
47. Stoeckius, M. et al. Simultaneous epitope and transcriptome measurement in single cells. Nat. Methods 14, 865-868 (2017).

48. Peterson, V. M. et al. Multiplexed quantification of proteins and transcripts in single cells. Nat. Biotechnol. 35, 936-939 (2017).

49. Luecken, M. D. \& Theis, F. J. Current best practices in single-cell RNA-seq analysis: a tutorial. Mol. Syst. Biol. 15, e8746 (2019).

50. Berger, B. \& Cho, H. Emerging technologies towards enhancing privacy in genomic data sharing. Genome Biol. 20, 128 (2019).

51. Wang, S., Pisco, A. O., Karkanias, J. \& Altman, R. B. Unifying single-cell annotations based on the Cell Ontology. bioRxiv 810234 (2019) doi:10.1101/810234.

52. Gayoso, A. et al. A Joint Model of RNA Expression and Surface Protein Abundance in Single Cells. bioRxiv 791947 (2019) doi:10.1101/791947.

53. Baglama, J. \& Reichel, L. Augmented Implicitly Restarted Lanczos Bidiagonalization Methods. SIAM Journal on Scientific Computing vol. 27 19-42 (2005).

54. Korsunsky, I., Nathan, A., Millard, N. \& Raychaudhuri, S. Presto scales Wilcoxon and auROC analyses to millions of observations. bioRxiv 653253 (2019) doi:10.1101/653253.

55. Friedman, J., Hastie, T. \& Tibshirani, R. Regularization Paths for Generalized Linear Models via Coordinate Descent. J. Stat. Softw. 33, 1-22 (2010).

56. Durinck, S., Spellman, P. T., Birney, E. \& Huber, W. Mapping identifiers for the integration of genomic datasets with the R/Bioconductor package biomaRt. Nat. Protoc. 4, 1184-1191 (2009).

57. Gu, Z., Eils, R. \& Schlesner, M. Complex heatmaps reveal patterns and correlations in multidimensional genomic data. Bioinformatics 32, 2847-2849 (2016).

58. Leurgans, S. E., Moyeed, R. A. \& Silverman, B. W. Canonical Correlation Analysis When the Data are Curves. J. R. Stat. Soc. Series B Stat. Methodol. 55, 725-740 (1993).

59. McInnes, L., Healy, J. \& Melville, J. UMAP: Uniform Manifold Approximation and Projection for Dimension Reduction. arXiv [stat.ML] (2018). 